\title{
A Census of B[e] Supergiants
}

\author{
Michaela Kraus \\ Astronomical Institute, Czech Academy of Sciences, Fričova 298, 25165 Ondřejov, Czech Republic; \\ michaela.kraus@asu.cas.cz
}

Received: 7 August 2019; Accepted: 25 September 2019; Published: 29 September 2019

\begin{abstract}
Stellar evolution theory is most uncertain for massive stars. For reliable predictions of the evolution of massive stars and their final fate, solid constraints on the physical parameters, and their changes along the evolution and in different environments, are required. Massive stars evolve through a variety of short transition phases, in which they can experience large mass-loss either in the form of dense winds or via sudden eruptions. The B[e] supergiants comprise one such group of massive transition objects. They are characterized by dense, dusty disks of yet unknown origin. In the Milky Way, identification and classification of B[e] supergiants is usually hampered by their uncertain distances, hence luminosities, and by the confusion of low-luminosity candidates with massive pre-main sequence objects. The extragalactic objects are often mistaken as quiescent or candidate luminous blue variables, with whom B[e] supergiants share a number of spectroscopic characteristics. In this review, proper criteria are provided, based on which B[e] supergiants can be unambiguously classified and separated from other high luminosity post-main sequence stars and pre-main sequence stars. Using these criteria, the $B[e]$ supergiant samples in diverse galaxies are critically inspected, to achieve a reliable census of the current population.
\end{abstract}

Keywords: stars: massive; stars: emission line, Be; supergiants; stars: winds, outflows; circumstellar matter

\section{Introduction}

Massive stars play a major role in the evolution of their host galaxies. Via stellar winds, they strongly enrich the interstellar medium (ISM) with chemically processed material and deposit large amounts of momentum and energy into their surroundings during their entire lifetime, from the main-sequence up to their final fate as spectacular supernova explosions (e.g., [1-3]). The released energy provides the ionizing radiation, substantially supplies the global energy budget of the host galaxy and significantly contributes to shaping the local ISM, whereas the released material condenses into molecules and dust, providing the cradles for the next generation of stars and planets (e.g., [4,5]).

Despite their great importance, stellar evolution theory is most uncertain for massive stars due to the often still poor understanding of some physical processes in the stellar interiors (e.g., core convective overshooting, chemical diffusion, internal differential rotation law and angular momentum transport), the excitation and propagation of pulsation instabilities within their atmospheres, the amount of mass loss via stellar (often asymmetric) winds and (irregular) mass ejections, and the role of binarity for certain phases.

From an observational point of view, the post-main sequence domain within the Hertzsprung-Russell (HR) diagram is populated with various types of extreme massive stars. These are found to be in transition phases, in which the stars shed huge amounts of material into their environments, typically via episodic, sometimes even eruptive events. These objects are luminous super- or hypergiants populating the upper part of the HR diagram and spreading from spectral type $\mathrm{O}$ to $\mathrm{F}$ or even later. The ejected material thereby accumulates in either nebulae, shells, or even disk-like structures. 
The mass-loss of massive stars not only critically depends on the physical parameters, such as mass, effective temperature, and rotation speed, but also on the chemical composition of the star. The amount of mass that is lost, within each individual evolutionary phase, determines the fate of the object. It is thus not surprising that relative numbers of various types of massive stars can change drastically among galaxies with different metallicities (e.g., [6]). For reliable predictions of the evolutionary path of massive stars in any environment, solid constraints on the physical parameters, used in modern stellar evolution models, are indispensable. To obtain such constraints, the properties of the members within each class of objects need to be studied in great detail and within a variety of environments. This requires statistically significant samples of stars in each class of objects, suitable for a detailed analysis. The star-forming galaxies within the local Universe, in which the metallicities spread over a factor of about 25 between the most metal poor and the most metal rich representative, are the most ideal sites to tackle this challenge.

This review is devoted to the $\mathrm{B}[\mathrm{e}]$ supergiants, which comprise one of the various classes of extreme massive stars in transition. The article is structured as follows. First, an overview on the general properties of these stars is given based mostly on the well-studied sample within the Magellanic Clouds (Section 2), followed by a review on how these objects are searched for in various environments (Section 3). A census of the currently known objects in the Local Group galaxies and slightly beyond is presented in Section 4, based on a critical inspection of the properties of the individual candidates. The discussion of the B[e]SG samples and our conclusions are finally summarized in Section 5.

\section{B[e] Supergiants}

The early-type supergiants include a class of emission-line objects, whose optical spectra display a peculiar character with strong Balmer emission along with narrow emission lines from permitted and forbidden transitions (e.g., [7-10]). The latter are indicative of a cool and slowly expanding medium. With the advent of ultraviolet (UV) observations taken with the International Ultraviolet Explorer (IUE), these stars were found to display very broad blueshifted resonance lines of highly ionized elements in this spectral range. These resonance lines originate from a hot and fast stellar line-driven wind which is very typical for supergiants in this temperature and luminosity range.

Another peculiar property of these stars was discovered in the near-infrared, in which these objects possess a pronounced excess emission pointing to hot circumstellar dust [11-16]. This dust was proposed to be most likely produced within the slow and cool component and to possibly populate a ring or disk-like region at far distances from the luminous central objects [15].

In the HR diagram, these objects are all found beyond the main-sequence and with luminosities spreading from about $\log L / L_{\odot} \sim 4$ to about $\log L / L_{\odot} \sim 6$, implying that they are all evolved, massive stars. This luminosity range was determined from the sample residing in the Magellanic Clouds (MCs), for which the luminosity determination is unquestionable, due to the low extinction towards the MCs and their well constraint distances. The classification of Galactic objects as supergiants bears much higher uncertainties due to their often poorly constrained distances, hence luminosities. We come back to this issue in Section 4.4.

The position of the MC sample in the HR diagram is shown in Figure 1 for the values of luminosity and effective temperature listed in Table 1 . The stellar parameters (effective temperature

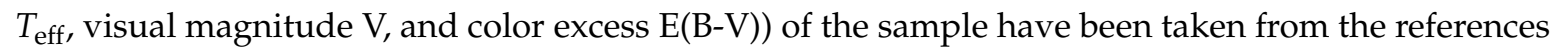
listed in the last column of Table 1. For the calculations of the luminosities, distance moduli of 18.5 and 18.9 mag, respectively, for the Large and Small Magellanic Clouds have been utilized (see the review paper by Humphreys, this volume) along with bolometric corrections from [17]. 


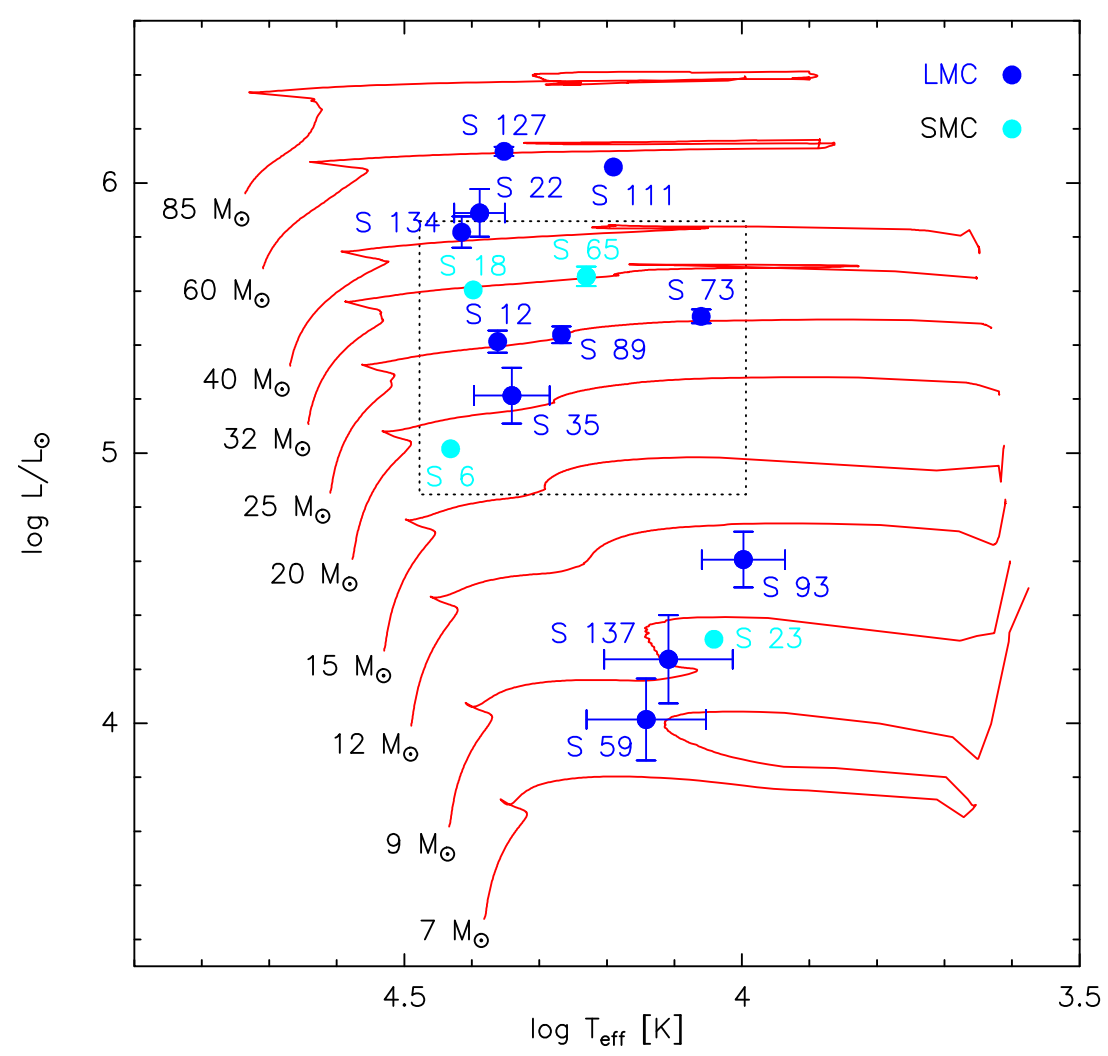

Figure 1. HR diagram showing the positions of the classical MC B[e]SG sample [18]. The stellar evolutionary tracks at SMC metallicity for stars rotating initially with $40 \%$ of their critical velocity are also included (from [19]). The dotted square contains objects that display CO band emission (except for S 89, see Section 2.3). For brevity and readability, the identifiers LHA 120 and LHA 115 for objects within the LMC and SMC, respectively, have been omitted.

Table 1. Names and parameters of the established B[e]SG sample within the Magellanic Clouds.

\begin{tabular}{|c|c|c|c|c|c|c|}
\hline Object & Other Common Identifiers & $\log T_{\text {eff }}$ & $\log L / L \odot$ & $\mathbf{V}$ & $\mathrm{E}(\mathrm{B}-\mathrm{V})$ & Ref. \\
\hline \multicolumn{7}{|c|}{ Large Magellanic Cloud B[e]SGs } \\
\hline LHA $120-S 12$ & SK -6723 & 4.36 & $5.41 \pm 0.04$ & 12.6 & $0.2-0.25$ & [16] \\
\hline LHA 120-S 22 & HD 34664, SK -67 64 & $4.39 \pm 0.04$ & $5.89 \pm 0.09$ & 11.7 & $0.25-0.3$ & [16] \\
\hline LHA 120-S 35 & SK -6697 & $4.34 \pm 0.06$ & $5.21 \pm 0.10$ & 12.5 & 0.07 & [20] \\
\hline LHA 120-S59 & $\ldots$ & $4.14 \pm 0.09$ & $4.01 \pm 0.15$ & 14.4 & 0.05 & [20] \\
\hline LHA 120-S 73 & RMC 66, HD 268835 & 4.06 & $5.51 \pm 0.03$ & 10.6 & $0.12-0.15$ & [13] \\
\hline LHA 120-S 89 & RMC 82, HD 269217 & 4.27 & $5.44 \pm 0.03$ & 12.0 & 0.20 & [16] \\
\hline LHA 120-S 93 & SK -6866 & $4.00 \pm 0.06$ & $4.61 \pm 0.10$ & 12.7 & 0.22 & [20] \\
\hline LHA $120-S 111^{\text {a }}$ & HD 269599 & 4.19 & 6.06 & 10.3 & 0.28 & [21] \\
\hline LHA 120-S 127 & RMC 126, HD 37974 & 4.35 & $6.12 \pm 0.02$ & 10.9 & 0.25 & [15] \\
\hline LHA $120-S 134$ b,c & HD 38489, SK -69 259 & 4.42 & $5.82 \pm 0.06$ & 12.0 & $0.2-0.25$ & [16] \\
\hline LHA 120-S 137 & $\ldots$ & $4.11 \pm 0.10$ & $4.24 \pm 0.16$ & 14.0 & 0.17 & [20] \\
\hline \multicolumn{7}{|c|}{ Small Magellanic Cloud B[e]SGs } \\
\hline LHA $115-S 6^{b}$ & $\mathrm{RMC} 4, \mathrm{AzV} 16$ & 4.43 & 5.02 & 13.0 & 0.07 & [22] \\
\hline LHA $115-S 18$ b,c & AzV 154 & 4.40 & 5.60 & 13.3 & 0.4 & [23] \\
\hline LHA 115-S 23 & $\mathrm{AzV} 172$ & 4.04 & 4.31 & 13.3 & $0.03-0.1$ & {$[24]$} \\
\hline LHA 115-S 65 & RMC 50 & 4.23 & $5.65 \pm 0.04$ & 11.6 & $0.15-0.2$ & [16] \\
\hline
\end{tabular}

Note: Former designations of some of the objects as Hen S\# (see, e.g., [25]) were omitted here, as these are not SIMBAD identifiers. However, LHA 120-S and LHA 115-S, respectively, and the former Hen $S$ numbers refer to the same objects. a The star is also listed as RMC 105 in SIMBAD, but this designation should be used for a neighboring, normal B-type star in this dense cluster (see [26]). ${ }^{b}$ Confirmed or suspected binary. ${ }^{c}$ X-ray source [27-29]. 
The presence of dust around an early-type (typically of spectral type B) supergiant, along with the often pure emission-line spectra with numerous forbidden lines predominantly of [Fe II] and [O I] finally resulted in the designation of these objects as B[e] supergiants (B[e]SGs) ${ }^{1}$.

\subsection{General Aspects of B[e]SG Stars' Disks}

There is compelling evidence that B[e]SGs are surrounded by gaseous and dusty disks. The simultaneous presence of a hot and fast polar wind traced in the UV, and the cool and slow equatorial wind traced at optical wavelengths led to the assignment of a so-called hybrid or two-component wind model [15]. For this two-component wind, a density contrast between the equatorial and polar components of 100-1000 was proposed, meaning that the equatorial wind might be assigned the character of an outflowing disk [23].

The degree of non-sphericity of the envelopes and the latitude dependence of the wind density, respectively, are pursued by the measured net intrinsic polarization [30,31] and from spectropolarimetric observations [32,33]. The often high degree of intrinsic polarization support the idea of a combination of Thomson scattering by free electrons and Mie scattering by dust in a circumstellar disk [34].

If the disks of B[e]SGs are supposed to form from a high-density equatorial stellar outflow, there should be a transition zone between the atomic gas and the location of the dust, in which molecules can form in substantial amounts, because the high gas density can shield the material from the direct irradiation with dissociating UV photons coming from the hot luminous star. In fact, molecular emission, in particular of the first-overtone bands of carbon monoxide (CO), has been detected in the K-band spectra of a number of B[e]SGs in the Galaxy and the MCs (e.g., [21,35-44], see Table 2). To produce the characteristic observed emission spectra with several individual band heads, temperatures of the $\mathrm{CO}$ gas higher than $\sim 2000 \mathrm{~K}$ are required. These temperatures are in excess of the dust sublimation temperature, which is on the order of $\sim 1500 \mathrm{~K}$, placing the $\mathrm{CO}$ emitting region closer to the star than the dust.

Additional hot molecular emission from silicon oxide $(\mathrm{SiO})$ has been identified in four Galactic B[e]SGs [45], and a feature arising in the optical spectrum, which has been tentatively identified as emission from titanium oxide (TiO), was reported from six MC B[e]SGs [23,43,44,46]. However, to date, no systematic surveys for molecular emission has been performed, so that these numbers are not representative for the existence or absence of molecules in the environments of B[e]SGs. For instance, $\mathrm{SiO}$ emission has not been searched for yet in any of the MC B[e]SGs, and only those Galactic B[e]SGs with the most intense $\mathrm{CO}$ band emission have been observed in the wavelength range of the first-overtone band of $\mathrm{SiO}$ arising in the L-band. Hence, one might expect to find molecular emission from $\mathrm{SiO}$ in many more objects, but also emission from other yet undiscovered molecules that might form in the environments of B[e]SGs. What is interesting though is the fact that all MC stars displaying $\mathrm{TiO}$ emission also have $\mathrm{CO}$ emission, whereas the opposite does not hold. No detection of $\mathrm{TiO}$ from Galactic B[e]SGs has been reported so far.

Finally, the power of optical interferometry operating at near- and mid-infrared wavelengths should be mentioned when talking about the disks of B[e]SGs. Based on this technique, the disks of the closest and infrared brightest Galactic objects could be spatially resolved, providing precise measurements of the disk inclinations, disk sizes, and the distances of the emitting material (dust, $\mathrm{CO}$ gas, and ionized gas traced by the $\mathrm{Br} \gamma$ emission) from the central star (see [38,47-52]).

1 Note that these objects have previously been abbreviated sgB[e] [25], to separate them from other stars showing the B[e] phenomenon. We prefer the designation $B[\mathrm{e}] \mathrm{SG}$, to be in line with the naming and abbreviation of other types of supergiants such as blue supergiant (BSG) and red supergiant (RSG). 
Table 2. Presence of disk tracers in the optical and near-IR spectra of the Galactic and Magellanic Cloud B[e]SG samples.

\begin{tabular}{|c|c|c|c|c|c|c|c|c|c|}
\hline Object & [Ca II] & {$\left[\mathrm{O}_{\mathrm{I}}\right]^{\mathrm{a}}$} & Ref. & $\mathrm{CO}$ & ${ }^{12} \mathrm{C} /{ }^{13} \mathrm{C}$ & Ref. & $\mathrm{TiO}$ & $\mathrm{SiO}$ & Ref. \\
\hline \multicolumn{10}{|c|}{ Large Magellanic Cloud B[e]SGs } \\
\hline LHA $120-S 12$ & yes & no & [53] & yes & $20 \pm 2$ & {$[21,41]$} & yes & $\ldots$ & [23] \\
\hline LHA 120-S 22 & yes & yes & [53] & no & $\ldots$ & {$[21,41]$} & no & $\ldots$ & $\mathrm{TW}^{\mathrm{b}}$ \\
\hline LHA 120-S 35 & yes & yes & [44] & yes & $10 \pm 2$ & {$[41,44]$} & yes & $\ldots$ & [44] \\
\hline LHA 120-S 59 & no & yes & [54] & $?$ & $\ldots$ & [41] & no & $\ldots$ & $\mathrm{TW}^{\mathrm{b}}$ \\
\hline LHA 120-S 73 & yes & yes & {$[43,53]$} & yes & $9 \pm 1$ & {$[21,41,43]$} & yes & $\ldots$ & {$[55]$} \\
\hline LHA $120-S 89$ & no & no & $\mathrm{TW}^{\mathrm{b}}$ & no & $\ldots$ & {$[21,41]$} & no & $\ldots$ & $\mathrm{TW}^{\mathrm{b}}$ \\
\hline LHA 120-S 93 & yes & no & $\mathrm{TW}^{\mathrm{b}}$ & no & $\ldots$ & [41] & no & $\ldots$ & $\mathrm{TW}^{\mathrm{b}}$ \\
\hline LHA 120-S111 & yes & yes & [53] & no & $\ldots$ & [21] & yes & $\ldots$ & {$[23]$} \\
\hline LHA $120-S 127$ & yes & yes & {$[53,56]$} & no & $\ldots$ & {$[21,41]$} & no & $\ldots$ & $\mathrm{TW}^{\mathrm{b}}$ \\
\hline LHA 120-S 134 & yes & yes & [53] & yes & $15 \pm 2$ & {$[21,41]$} & yes & $\ldots$ & [23] \\
\hline LHA 120-S 137 & no & yes & $\mathrm{TW}^{\mathrm{b}}$ & no & $\ldots$ & [41] & no & $\ldots$ & $\mathrm{TW}^{\mathrm{b}}$ \\
\hline \multicolumn{10}{|c|}{ Small Magellanic Cloud B[e]SGs } \\
\hline LHA 115-S 6 & $?$ & yes & $\mathrm{TW}^{\mathrm{b}}$ & yes & $12 \pm 2$ & {$[36,41]$} & no & $\ldots$ & $\mathrm{TW}^{\mathrm{b}}$ \\
\hline LHA 115-S 18 & yes & yes & [53] & yes & $20 \pm 5$ & {$[37,41]$} & yes & $\ldots$ & {$[23,46]$} \\
\hline LHA $115-S 23^{c}$ & $?$ & no & $\mathrm{TW}^{\mathrm{b}}$ & no & $\ldots$ & $\mathrm{TW}^{\mathrm{d}}$ & no & $\ldots$ & $\mathrm{TW}^{\mathrm{b}}$ \\
\hline LHA 115-S 65 & yes & yes & {$[53,57]$} & yes $^{\mathrm{e}}$ & $20 \pm 5$ & {$[40,41]$} & no & $\ldots$ & $\mathrm{TW}^{\mathrm{b}}$ \\
\hline \multicolumn{10}{|c|}{ Galactic B[e]SGs/B[e]SG Candidates } \\
\hline MWC 137 & no & no & {$[55,58]$} & yes & $25 \pm 2$ & {$[39,41,59]$} & no & $\ldots$ & $\mathrm{TW}^{\mathrm{b}}$ \\
\hline MWC 349 & yes & $\ldots$ & {$[60]$} & yes & $4 \pm 1$ & {$[61,62]$} & $\ldots$ & no & {$[62]$} \\
\hline GG Car & yes & no & [58] & yes & $15 \pm 5$ & {$[35,37,39,41,42,58]$} & no & $\ldots$ & $\mathrm{TW}^{\mathrm{b}}$ \\
\hline Hen 3-298 & yes & yes & {$[58,63]$} & yes & $20 \pm 5$ & {$[39,41,58,63]$} & no & $\ldots$ & $\mathrm{TW}^{\mathrm{b}}$ \\
\hline CPD-52 9243 & yes & no & [58] & yes & $\ldots$ & {$[35,39,58]$} & no & yes & $\mathrm{TW}^{\mathrm{b}},[45]$ \\
\hline HD 327083 & yes & no & [58] & yes & $\ldots$ & {$[39,58]$} & no & yes & $\mathrm{TW}^{\mathrm{b}},[45]$ \\
\hline MWC 300 & no & yes & $\mathrm{TW}^{\mathrm{b}}$ & no & $\ldots$ & {$[39,64]$} & no & $\ldots$ & $\mathrm{TW}^{\mathrm{b}}$ \\
\hline AS 381 & no & no & [65] & abs & $?$ & {$[39,64,65]$} & $\ldots$ & $\ldots$ & $\ldots$ \\
\hline CPD-57 2874 & yes & no & [58] & yes & $\ldots$ & {$[35,39,58]$} & no & yes & $\mathrm{TW}^{\mathrm{b}},[45]$ \\
\hline Hen 3-938 & yes & yes & [54] & $\ldots$ & $\ldots$ & $\ldots$ & no & $\ldots$ & $\mathrm{TW}^{\mathrm{b}}$ \\
\hline MWC 342 & no & yes & {$[60,66]$} & $\ldots$ & $\ldots$ & $\ldots$ & $\ldots$ & $\ldots$ & $\ldots$ \\
\hline Hen 3-303 & no & no & $\mathrm{TW}^{\mathrm{b}}$ & no & $\ldots$ & [63] & no & $\ldots$ & $\mathrm{TW}^{\mathrm{b}}$ \\
\hline CD-42 11721 & no & yes & [67] & no & $\ldots$ & [39] & no & $\ldots$ & $\mathrm{TW}^{\mathrm{b}}$ \\
\hline HD 87643 & yes & no & [58] & yes $^{e}$ & $\ldots$ & {$[39,58]$} & no & $\ldots$ & $\mathrm{TW}^{\mathrm{b}}$ \\
\hline $\operatorname{HD} 62623^{c}$ & yes & yes & [58] & yes & $\ldots$ & {$[39,58]$} & no & yes & $\mathrm{TW}^{\mathrm{b}},[45]$ \\
\hline
\end{tabular}

Note: TW, This work; abs, in absorption; ?, uncertain detection/no value available; ..., no information. a Refers to the presence of [O I] $\lambda 5577$. All sample stars show emission of $[\mathrm{O} I] \lambda \lambda 6300,6364$. $^{\mathrm{b}}$ Based on (unpublished) high-resolution optical spectra taken between 2005 and 2017 with FEROS at the MPG 2.2 m telescope. ${ }^{c}$ A[e]SG due to early-A spectral type assignment $[68,69]$. d No indication of CO band features seen in a K-band spectrum (unpublished) taken on 20 October 2013 with OSIRIS at the Southern Astrophysical Research (SOAR) Telescope. ${ }^{\mathrm{e}} \mathrm{No} \mathrm{CO}$ emission was detected during the observations taken between 1987 and $1989[35,36]$.

\subsection{Disk Dynamics and Structure}

Determination of the kinematics within dense circumstellar environments requires the use of reliable tracers. High-resolution near-infrared spectroscopic observations have revealed that the band heads of the CO emission from B[e]SGs typically display a characteristic shape, consisting of a blue-shifted shoulder and a red-shifted maximum. For the generation of such a band head profile, the individual CO rotation-vibration lines, superimposing within the region of the band head, must display double-peaked profiles (see Figure 2). Such line profiles can originate either from a circumstellar ring of gas expanding with constant velocity (constant outflow), or from rotational motion of a ring of gas around the central object. To discriminate between the two scenarios, complementary tracers are needed. 


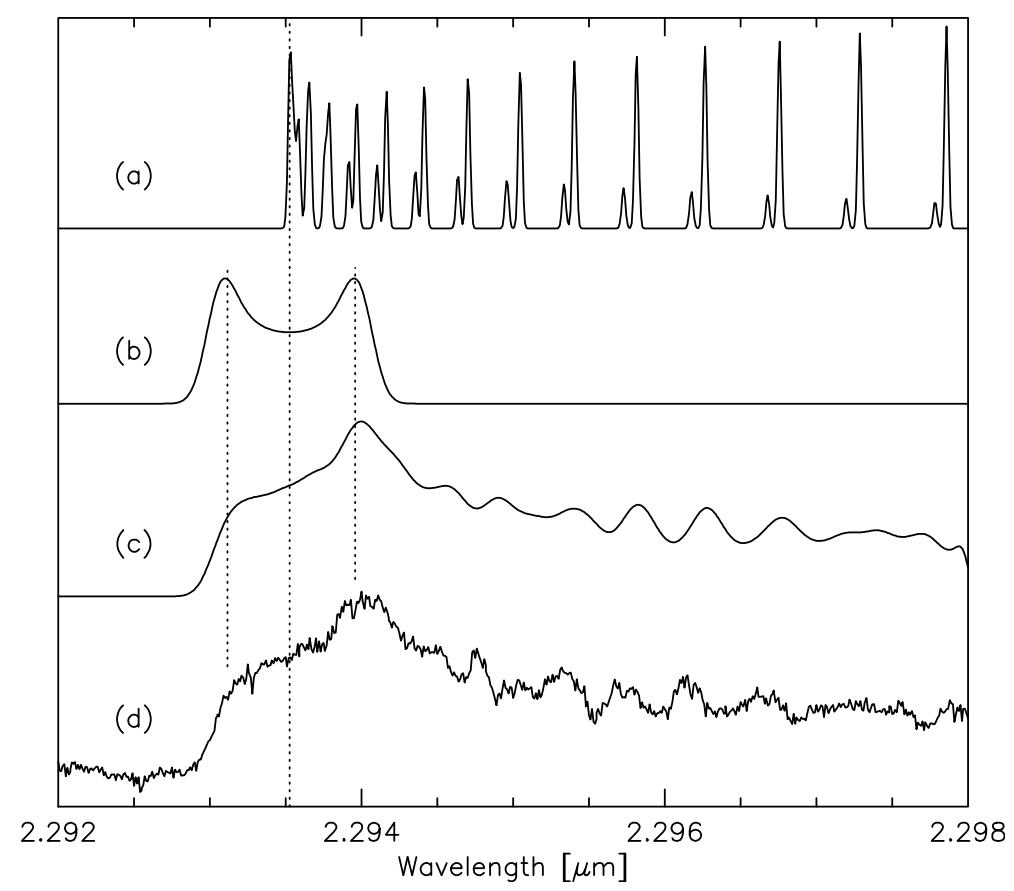

Figure 2. Sketch of the generation of the typical CO band head profile. (a) Spectrum around the (2-0) band head of the CO first-overtone bands for a hot gas with velocity dispersion of a few $\mathrm{km} \mathrm{s}^{-1}$. (b) Profile of a single line from a rotating gas ring with a velocity, projected to the line of sight, of $66 \mathrm{~km} \mathrm{~s}^{-1}$ as seen with a spectral resolution of $6 \mathrm{~km} \mathrm{~s}^{-1}$. (c) Total synthetic CO band head spectrum resulting from the convolution of the band transitions in (a) with the profile of the ring in (b). (d) $\mathrm{CO}$ band head observations of the Galactic B[e]SG CPD-57 2874 [58].

The $\mathrm{SiO}$ band emission seen in four Galactic B[e]SGs displays a similar shape of the band heads. Detailed modeling revealed that in each object the $\mathrm{SiO}$ bands required a slightly lower value of the velocity [45] than the $\mathrm{CO}$ bands. The $\mathrm{SiO}$ molecule is less stable than $\mathrm{CO}$, meaning that it can form and persist only at lower temperatures. This fact naturally places the region where $\mathrm{SiO}$ molecules are expected to form, and hence the $\mathrm{SiO}$ band emitting region, at slower orbital velocities and greater distances from the central object.

As $\mathrm{CO}$ is the most stable molecule, its formation and emission region marks the inner edge of the molecular disk. Closer to the star, tracers for the kinematics need to be found from line emission of the atomic gas. Here, the lines from forbidden transitions are most suitable, because their emission is optically thin, so that their profile shapes contain the full velocity information of their formation region [56,57]. Of particular interest are hereby the [O I] lines, because they are one of the defining characteristics of the $\mathrm{B}[\mathrm{e}]$ phenomenon and hence observed in all $\mathrm{B}[\mathrm{e}] \mathrm{SG}$. The ionization potential of $\mathrm{O}$ I is about the same as the one for $\mathrm{HI}$, which means that within the [O I] line forming regions, hydrogen should be basically neutral as well, restricting the formation region of the [O I] line emission to the neutral regions within the circumstellar disk. While recombination in the equatorial region close to the star might be achieved, e.g., with the model of a latitude dependent wind [70-72], the requirement of a hydrogen neutral environment severely limits the number of free electrons that will be available to collisionally excite the levels within $\mathrm{O}$ I from which the forbidden transitions emerge. Consequently, the [O I] lines arise in regions with high total density, but low electron density.

The profiles of the [O I] lines often display double-peaks, in line with their formation in the disk. Typically, the [O I] $\lambda 5577$ line, which arises from a higher level than the $\lambda \lambda 6300,6364$ lines, is broader, indicating spatially distinct formation regions of the emissions, with the [O I] $\lambda 5577$ line being formed at higher velocities and higher densities and hence closer to the star than the other two lines.

With the identification of the lines of [Ca II] $\lambda \lambda 7291,7324$ in the spectra of numerous B[e]SGs, a further highly valuable tracer for the disk kinematics has been found. These lines typically display 
double-peaked profiles as well, with velocities comparable to or even greater than the one traced by the [O I] $\lambda 5577$ line $[53,57,58]$. This implies that they form in the same region, or at least very close to each other, which is in agreement with their comparably high critical density. Since the [O I] $\lambda 5577$ line is not always detectable, the [Ca II] lines thus provide a suitable, complementary benchmark for the dynamics within the disks of B[e]SGs.

In summary, the optical and near-infrared spectra provide emission features from several species, which are suitable to pin down the kinematics within the disks of B[e]SGs at various distances from the star, and Table 2 includes the information on the detection of the individual tracers in the MC sample. Based on the physical constraints outlined above, the logical order of the appearance of the divers tracers from inside out would be: [Ca II], [O I], $\mathrm{CO}$ bands and $\mathrm{SiO}$ bands. The velocity information carried by these species thereby implies a decrease with increasing distance from the star. While an equatorial, outwards decelerating outflow might be able to explain some of the observed line profiles [56], the velocity patterns seem to be in better agreement with (quasi-)Keplerian rotation. In this respect, it is interesting to note that Keplerian rotation has been made directly discernible by means of spectro-interferometric observations. The rotational motion of the $\mathrm{CO}$ gas has been derived based on the differential phase spectrum [38,52], and the rotational motion of the ionized gas based on the spatially resolved $\mathrm{Br} \gamma$ emission [49].

While, in general, the rotational motion of the material within the circumstellar disks of B[e]SGs seems now to be well established, possibly in connection with a (very) slow outflow component [57], recent investigations of the spatial distribution of the circumstellar gas revealed that it is more likely accumulated in multiple rings, partial rings, and possible spiral arm-like structures rather than in a smooth disk $[43,44,58]$. These rings might result from multiple mass ejection phases caused by (pulsational) instabilities acting in the outer layers of these luminous objects (e.g., [73-75]), or from binary interaction in close systems, as seems to be the case for some of the Galactic objects [58], in which the rings are circumbinary rather than circumstellar. Other disk-forming mechanisms that have been proposed in the literature over the years include equatorial mass-loss from a critically rotating star [76,77], the rotationally induced bi-stability mechanism [78], the slow-wind solution [79], and the combination of the latter two [80]. For an overview including a detailed description of the various models and their limitations, see [81].

The circumstellar material of many MC objects appears durable. This is evidenced by their emission features and their infrared photometry that both display no considerable variability over several decades, in combination with chemically processed dust displaying emission from crystalline silicates [82]. It is tempting to imagine that in such an environment even minor bodies might have formed from the long-lived disk material, creating gaps within the disk in radial direction and hence leading to the formation of the presumed ring structures [43]. These minor bodies or possible planets can also stabilize the neighboring rings, in analogy to the shepherd moons in planetary systems. However, thus far, there is insufficient observational evidence that might support the validity of such a scenario.

\subsection{Current Evolutionary State of B[e]SGs}

The formation mechanism of the observed gaseous and dusty rings or disk-like structures around $\mathrm{B}$ [e]SGs is certainly one of the most important yet unsolved issues. Equally important questions arise: What is the evolutionary phase of B[e]SGs? What is their evolutionary connection to other evolved massive stars? Does such a connection exist at all? While the question on the relation between $\mathrm{B}[\mathrm{e}] \mathrm{SGs}$ and other evolved objects is beyond the scope of this review, we briefly elucidate the current knowledge about the evolutionary status of B[e]SGs. Considering the MC sample, it is obvious from Figure 1 that all objects have evolved off the main sequence. Whether this occurred only recently, or whether B[e]SGs might be on a blue loop or blueward evolution after having passed through the turning point on the cool edge of their track, is still an open issue, in particular, since we lack clear 
methods for age determinations of these emission-line objects, which only very rarely are detected in clusters ${ }^{2}$.

In this regard, the detection of clear signs of the ${ }^{13} \mathrm{C}$ isotope in the form of ${ }^{13} \mathrm{CO}$ band emission from a number of MC B[e]SGs $([41,86]$, see Table 2) was one major step forward. The appearance of these bands has been predicted based on theoretical model computations for a variation of the carbon isotope ratio ${ }^{12} \mathrm{C} /{ }^{13} \mathrm{C}$ [87]. As surface abundance calculations have shown, this ratio will drop during the evolution of massive stars from an initial, interstellar value of $\sim 90$ down to values $<5$, depending on the initial mass of the star and its initial rotation speed. The surface material enriched in ${ }^{13} \mathrm{C}$ is transported via winds to the environments, where it will cool and condense into ${ }^{13} \mathrm{CO}$ molecules, whose emission can be observed in the K-band, together with the emission from the main isotope, ${ }^{12} \mathrm{CO}$ (see Figure 3). Hence, the detected amount of ${ }^{13} \mathrm{CO}$ is a measure for the stellar surface enrichment in ${ }^{13} \mathrm{C}$ at the time the material, which is currently traced in the molecular emission, has been released from the stellar surface.

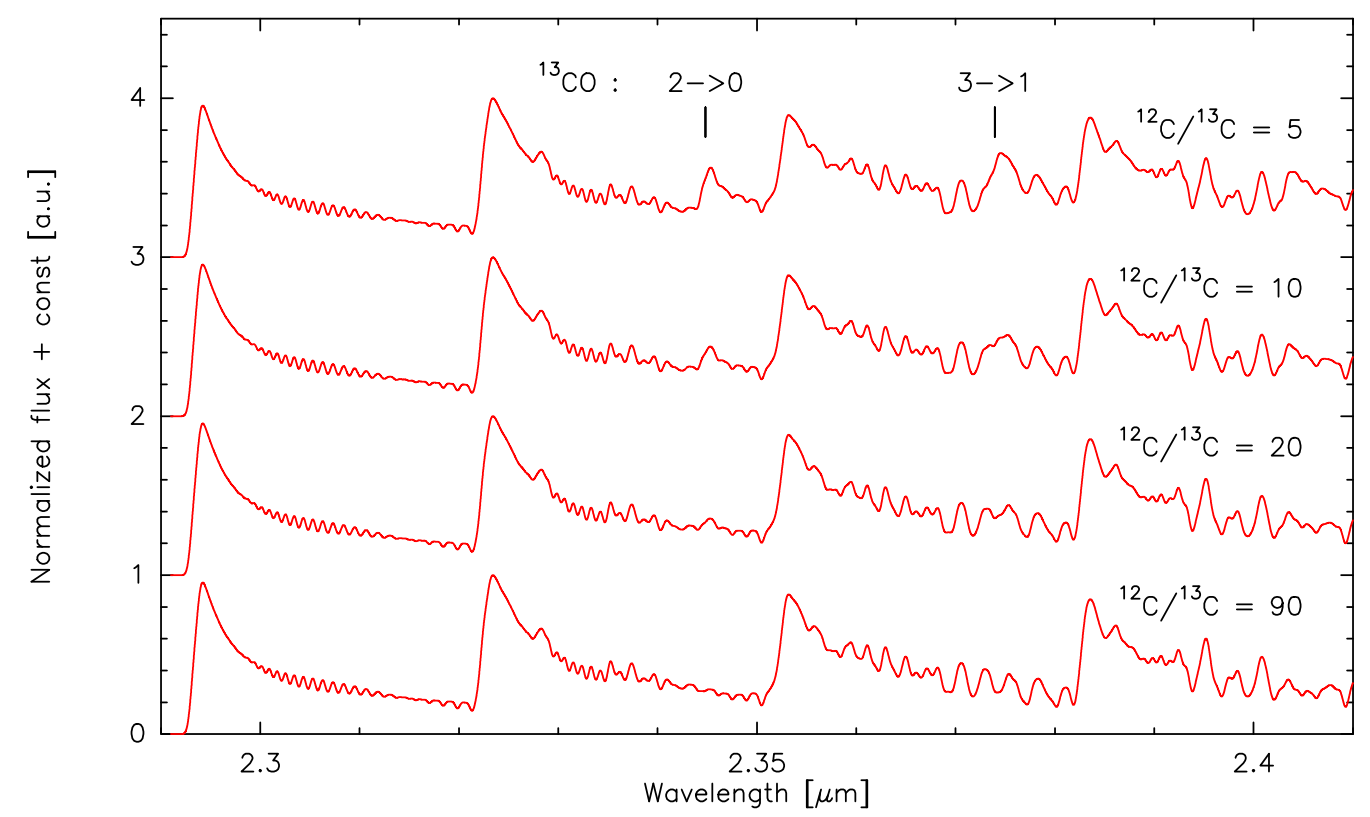

Figure 3. Synthetic spectra of the combined emission from ${ }^{12} \mathrm{CO}$ and ${ }^{13} \mathrm{CO}$ for different values of the ${ }^{12} \mathrm{C} /{ }^{13} \mathrm{C}$ ratio. The computations have been performed for the following physical parameters: a ${ }^{12} \mathrm{CO}$ column density of $2 \times 10^{21} \mathrm{~cm}^{-2}$, a gas temperature of $3000 \mathrm{~K}$, a line-of-sight rotational velocity of $66 \mathrm{~km} \mathrm{~s}^{-1}$, and a spectral resolution of $50 \mathrm{~km} \mathrm{~s}^{-1}$.

From the MC sample of 15 objects, seven have been found to display $\mathrm{CO}$ band emission, and all of them display clear indication of enrichment in ${ }^{13} \mathrm{C}$ (see Table 2). Interestingly, all these objects with $\mathrm{CO}$ emission cluster in the same region of the HR diagram, as indicated by the dotted black square in Figure 1, i.e., in the luminosity range $\log L / L_{\odot}=5.0-5.8$. None of the three most luminous stars (S22, S111, and S127) or of the four low-luminosity objects (S 23, S59, S 93, and S137) displays clear signs of $\mathrm{CO}$ emission. One outlier in the luminosity domain occupied by the CO emitting B[e]SGs is the star S 89, which also has no detectable CO band emission [41].

The absence of measurable $\mathrm{CO}$ band emission might have different reasons. Either the intensity of the emission is too low to be detectable against the strong near-IR continuum ${ }^{3}$, or the density of

2 Currently, only four B[e]SGs are reported to be cluster members: the two LMC objects LHA 120-S111 in the compact cluster NGC 1994 [26] and LHA 120-S35 in SL482 [44], and the two Galactic sources MWC 137 in SH2-266 [83] and Wd1-9 in Westerlund $1[84,85]$.

3 This spectral region suffers from strong telluric contamination, which is not always easy to remove, so that especially weak $\mathrm{CO}$ emission features might be hidden within telluric remnants. 
the molecular gas might be very high, resulting in optically thick emission, which no longer has a characteristic band head structure. Another possibility would be that the $\mathrm{CO}$ emission from these stars might have variable $\mathrm{CO}$ band emission and they have thus far always been observed in phases of no emission. In this context, it is interesting to refer to the SMC object LHA 115-S 65, in which CO band emission suddenly occurred, while observations taken about nine months earlier did not detect any molecular features [40]. Alternatively, since we now know that the material is most probably concentrated in rings, the conditions within the circumstellar environment in terms of density and temperature might not be favorable for the excitation of the first-overtone bands. For those stars, observations in the spectral region of the fundamental bands might therefore be a possibility to search for cooler CO gas.

The measured ${ }^{12} \mathrm{C} /{ }^{13} \mathrm{C}$ isotope ratios of the MC B[e]SGs are all very similar, spreading from 9 to 20. This might point towards a similar formation history of the circumstellar material, i.e., a similar phase in the evolution (considering they are single stars or at least unaffected by a possible companion) when the enriched material was ejected. Considering that stars are typically born with some intrinsic rotation velocity, rotational mixing in combination with enhanced mass-loss may drive the enrichment of the stellar surface with ${ }^{13} \mathrm{C}$ already in early stages of the evolution of massive stars. This can be seen in Figure 4, where the evolutionary tracks of a $32 \mathrm{M}_{\odot}$ star with solar metallicity [88] and for a variety of initial rotation velocities are shown. The covered rotation speeds spread from $v / v_{\text {crit }}=0$ to $v / v_{\text {crit }}=0.4$, which correspond to values of $\Omega / \Omega_{\text {crit }}$ from 0 to 0.568 . The interpolation of the tracks has been performed with the SCYCLIST tool ${ }^{4}$ provided by the Geneva group.

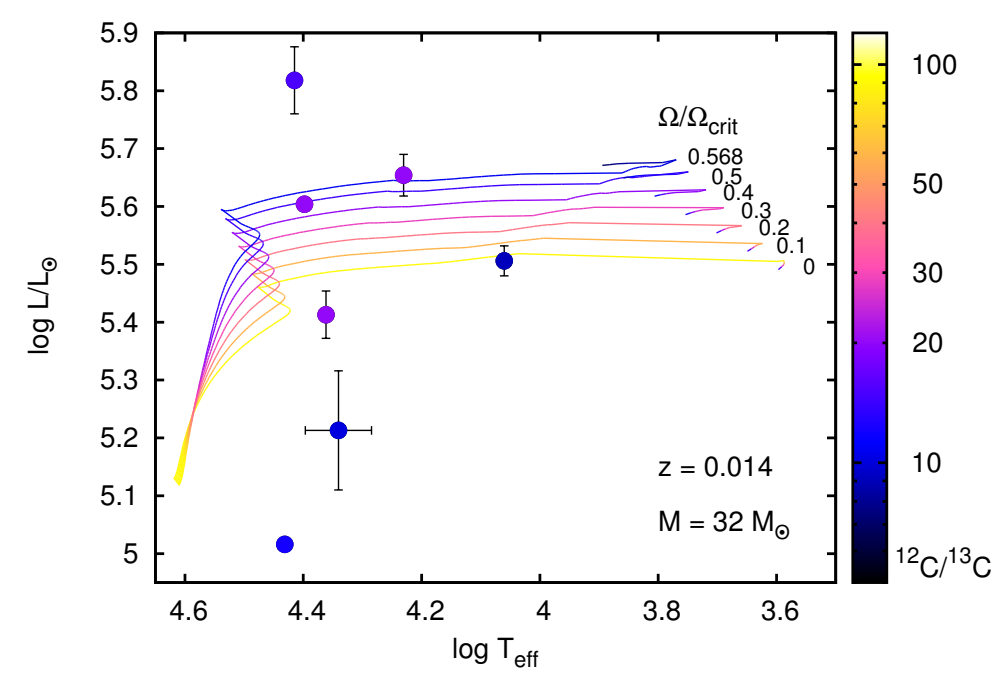

Figure 4. Evolution of the ${ }^{12} \mathrm{C} /{ }^{13} \mathrm{C}$ isotope ratio along the solar metallicity tracks of a star with initial mass of $32 \mathrm{M}_{\odot}$ and initial rotation speeds $v / v_{\text {crit }}$ ranging from 0 to 0.4 (corresponding to $\Omega / \Omega_{\text {crit }}=$ $0.0 ; 0.1 ; 0.2 ; 0.3 ; 0.4 ; 0.5 ; 0.568)$. The individual tracks have been obtained from the interpolation tool SCYCLIST provided by the Geneva group. For clarity of the plot, we truncated the evolutionary tracks within the red supergiant regions. Included are the positions of the MC B[e]SG sample from Table 2 with known ${ }^{12} \mathrm{C} /{ }^{13} \mathrm{C}$ ratio, following the same color coding as for the tracks. The Galactic objects are excluded due to their highly uncertain luminosities. Depending on the initial rotation speed of the star, the observed ratio can be reached either in the pre-RSG (moderate rotator) or post-RSG (slow rotator) phase.

The color coding along the tracks refers to the values of the ${ }^{12} \mathrm{C} /{ }^{13} \mathrm{C}$ isotope ratio on the stellar surface. Figure 4 also includes the positions of the seven MC B[e]SGs with known values of the 
${ }^{12} \mathrm{CO} /{ }^{13} \mathrm{CO}$ isotope ratios. Their colors correspond to the same color coding as the evolutionary tracks. Obviously, the observed ratios for the sample stars might be reached either along or after the main-sequence evolution for stars rotating initially with rates $\Omega / \Omega_{\text {crit }} \geq 0.3$. However, they might also be reached during or after the red supergiant stage for stars rotating initially with rates smaller than $\Omega / \Omega_{\text {crit }} \leq 0.3$. As we do not know the initial rotation speeds of the progenitor stars of these $\mathrm{B}[\mathrm{e}] \mathrm{SGs}$, the measured values of the ${ }^{12} \mathrm{CO} /{ }^{13} \mathrm{CO}$ isotope ratio alone cannot solve the issue with the current evolutionary state of the objects.

If B[e]SGs represent a specific phase in the evolution of massive stars, then these objects should also exist in other environments with high content of massive stars. Searching for representatives of B[e]SGs in other galaxies and studying their properties and number statistics at various metallicities might help to unveil their disk/ring formation mechanism, to pin down their evolutionary phase (preversus post-RSG), and to set constraints on the evolution of massive stars in general.

\section{Identification of $B[e]$ Supergiants in the Local Universe}

Identifying and classifying B[e]SGs is a tedious job, whether in the Milky Way, where we face large amounts of foreground extinction and the issue with the often unknown distances, or in other galaxies, where we have strong contamination with foreground sources, often crowded regions, and the faintness of the objects. While the first B[e]SGs have been found rather accidentally, nowadays dedicated surveys can make use of the established classification criteria. As mentioned in Section 2, there are basically four characteristics a star should fulfill to be classified as B[e]SGs $[18,25]$. It should display:

- A spectral-type B (with extensions to late-O and early-A types), as evidenced from the hot underlying continuum or from photospheric absorption features ${ }^{5}$, and a luminosity higher than $\log L / L_{\odot} \sim 4$.

- Very intense Balmer lines dominating the optical spectrum with an equivalent width of $\mathrm{H} \alpha$ reaching up to $1000 \AA$.

- A spectrum indicating the hybrid character of the environment, consisting of a hot, fast line-driven wind coexisting with a cool, slow component from which the forbidden emission lines from neutral and low-ionized metals originate ([O I], [Fe II]).

- $\quad$ Strong near- and mid-infrared excess emission, indicative of hot circumstellar dust.

Despite these defining criteria, the identification of extragalactic B[e]SGs is not as straightforward, because suitable candidates need first to be found based on other means. These candidates can then be further investigated to search for these, mostly spectroscopic, characteristics.

A suitable approach is to search for B[e]SG candidates among the luminous, blue objects identified in imaging surveys that have been performed for several galaxies over the past $\sim 30$ years. For instance, the early photographic and photometric surveys of M31 [89,90] and M33 [91] revealed (amongst many other objects) the most luminous hot stars and the brightest blue supergiants, which could then be studied spectroscopically to obtain indications for their possible nature (e.g., [92]). A milestone for the identification of evolved massive stars was certainly the Local Group Galaxies Survey (LGGS) project $[93,94]$, which resulted in the discovery of numerous putative emission-line stars in M31, M33, and seven more dwarf galaxies. Spectroscopic follow-ups were used to sort out $\mathrm{H}$ II regions, and to match the remaining objects with the various known categories of evolved massive stars. A major result of this survey was the identification of numerous objects that were dubbed as luminous blue variable (LBV) candidates [6,95], based on the appearance of their blue emission-line or P Cygni-type spectra that resemble confirmed LBVs in quiescence. Since the blue optical spectra of LBVs in their

5 We would like to stress that the high-luminosity B[e]SGs barely display photospheric absorption lines, whereas the low-luminosity B[e]SGs typically do. 
quiescence state display a number of common characteristics with B[e]SGs [95-97], it was expected that this sample contains a number of B[e]SGs.

Surprisingly, when analyzed in more detail [97-99], this bunch of newly identified LBV candidates in M31 and M33 turned out to be a mixed bag containing not only B[e]SGs candidates, but also so-called Fe II emission stars (with neither [O I] nor [Fe II] emission and lacking warm dust), and warm hypergiants (with lots of dust, possible [O I] and/or [Fe II] but of spectral type A-F), with only a few objects left to be considered as LBV candidates (with no [O I] emission and lacking hot dust).

As both LBVs and B[e]SGs are luminous blue supergiants, they share the same optical colors. Hence, one step to distinguish these two groups of objects is to inspect their location in infrared color-color diagrams (e.g., $[16,20,41,97,99])$. The hot $(\sim 1000 \mathrm{~K})$ circumstellar dust of B[e]SGs results in significantly increased near-IR emission. On the other hand, LBVs can be associated to cold, dusty environments such as the circumstellar shells recently discovered around many LBVs and LBV candidates with the Spitzer Space Telescope at $24 \mu \mathrm{m}$ ( e.g., [100,101]). The separation of B[e]SGs from quiescent LBVs, based on their diverse IR properties, is demonstrated in Figure 5 for the known samples of MC objects, limiting to the confirmed and generally accepted LBVs in the LMC [102] and including one confirmed object from the SMC (R40, [103]). Shown are two different color-color diagrams (J-H versus H-K and W1-W2 versus W2-W4). The IR colors of the objects are listed in Table 3. They result from the JHK-band magnitudes obtained from the 2MASS point source catalog ${ }^{6}$ [104], and from the mid-IR magnitudes (W1, W2, W4) collected with the Wide-field Infrared Survey Explorer ${ }^{7}$ (WISE [105]). From the many possibilities of near- and mid-IR color-color diagrams, these two show the clearest separation between the two groups of objects.
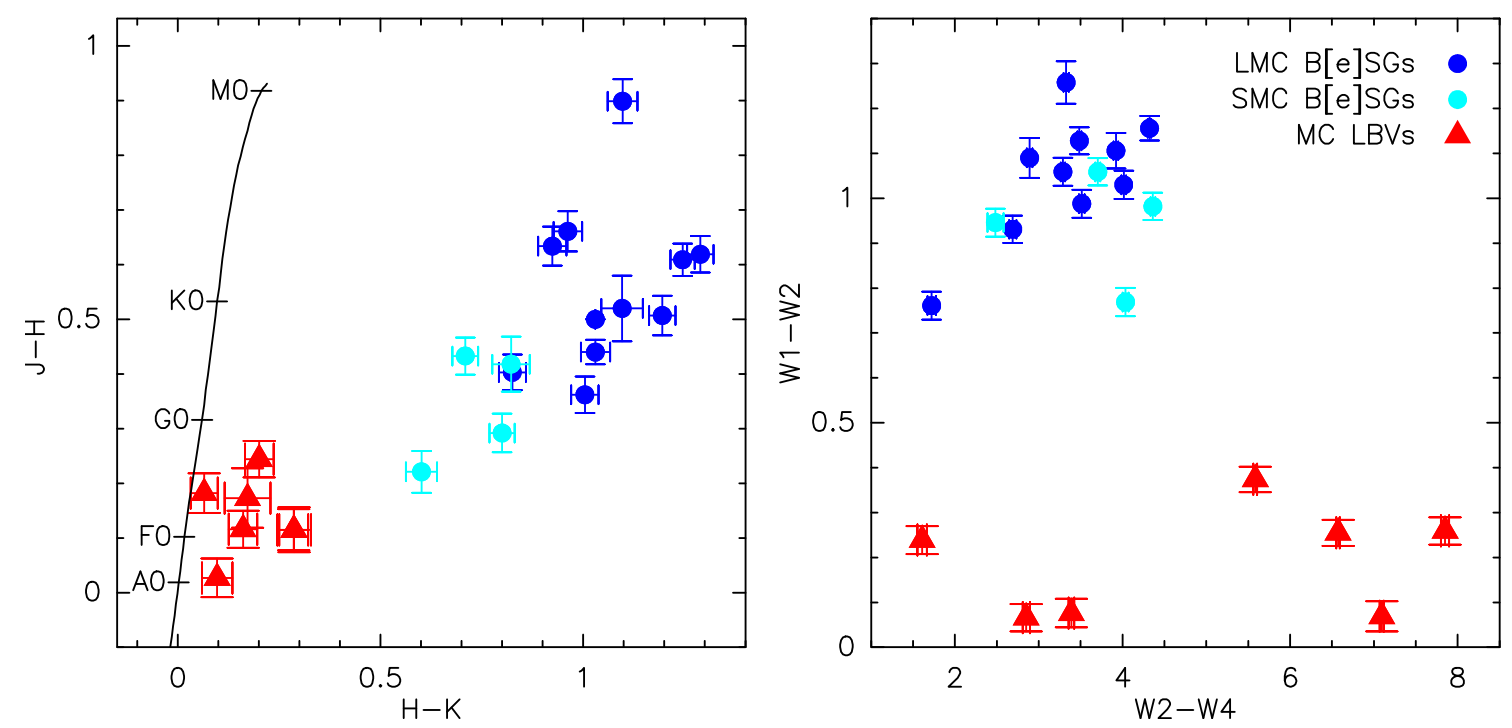

Figure 5. Demonstration of the separation of the B[e]SGs from the quiescent LBVs within the near-IR (J-H versus H-K diagram (left)) and the WISE diagram (W1-W2 versus W2-W4 (right)). Shown are the positions of the classical MC B[e]SG sample and of the MC LBV sample. IR colors of the objects are provided in Table 3. The solid line represents the positions of regular supergiants with empirical colors taken from [106] for solar metallicity stars.

Another clear distinctive feature between B[e]SGs and LBVs is the SDor cycle of the latter, while B[e]SGs are typically not undergoing this type of variability. However, to identify such SDor excursions of the stars within the HR diagram is a time-consuming (though important) task, because it requires regular monitoring of the whole sample. 
In addition, dedicated spectroscopic observations are required to search for the characteristic forbidden emission lines of [O I] and possible [Ca II] in the red portion of the optical spectra, and to search for possible molecular emission of CO in their near-IR spectra, because LBVs typically do not show these sets of forbidden lines and CO band emission ${ }^{8}[21,35,41]$.

Table 3. IR colors of Magellanic Cloud B[e]SGs and LBVs.

\begin{tabular}{|c|c|c|c|c|c|}
\hline Object & $\mathrm{J}-\mathrm{H}$ & H-K & W1-W2 & W2-W4 & Class \\
\hline \multicolumn{6}{|c|}{ Large Magellanic Cloud } \\
\hline LHA $120-S 12$ & $0.634 \pm 0.035$ & $0.924 \pm 0.035$ & $0.761 \pm 0.031$ & $1.723 \pm 0.051$ & $\mathrm{~B}[\mathrm{e}] \mathrm{SG}$ \\
\hline LHA $120-S 22$ & $0.619 \pm 0.033$ & $1.289 \pm 0.033$ & $1.258 \pm 0.047$ & $3.328 \pm 0.032$ & $\mathrm{~B}[\mathrm{e}] \mathrm{SG}$ \\
\hline LHA $120-S 35$ & $0.520 \pm 0.060$ & $1.096 \pm 0.051$ & $1.156 \pm 0.027$ & $4.323 \pm 0.021$ & $\mathrm{~B}[\mathrm{e}] \mathrm{SG}$ \\
\hline LHA 120-S 59 & $0.661 \pm 0.037$ & $0.962 \pm 0.035$ & $0.988 \pm 0.031$ & $3.514 \pm 0.033$ & $\mathrm{~B}[\mathrm{e}] \mathrm{SG}$ \\
\hline LHA $120-S 73$ & $0.403 \pm 0.033$ & $0.825 \pm 0.033$ & $1.030 \pm 0.031$ & $4.015 \pm 0.027$ & $\mathrm{~B}[\mathrm{e}] \mathrm{SG}$ \\
\hline LHA $120-S 89$ & $0.440 \pm 0.022$ & $1.030 \pm 0.036$ & $1.059 \pm 0.031$ & $3.289 \pm 0.026$ & $\mathrm{~B}[\mathrm{e}] \mathrm{SG}$ \\
\hline LHA 120-S 93 & $0.507 \pm 0.036$ & $1.195 \pm 0.033$ & $1.128 \pm 0.030$ & $3.486 \pm 0.025$ & $\mathrm{~B}[\mathrm{e}] \mathrm{SG}$ \\
\hline LHA $120-S 111^{a}$ & $0.500 \pm 0.000$ & $1.030 \pm 0.000$ & & & $\mathrm{~B}[\mathrm{e}] \mathrm{SG}$ \\
\hline LHA 120-S 127 & $0.362 \pm 0.033$ & $1.004 \pm 0.033$ & $1.106 \pm 0.039$ & $3.922 \pm 0.028$ & $\mathrm{~B}[\mathrm{e}] \mathrm{SG}$ \\
\hline LHA 120-S 134 & $0.609 \pm 0.030$ & $1.245 \pm 0.030$ & $1.090 \pm 0.045$ & $2.892 \pm 0.035$ & $\mathrm{~B}[\mathrm{e}] \mathrm{SG}$ \\
\hline LHA $120-S 137$ & $0.899 \pm 0.040$ & $1.097 \pm 0.037$ & $0.931 \pm 0.030$ & $2.691 \pm 0.045$ & $\mathrm{~B}[\mathrm{e}] \mathrm{SG}$ \\
\hline LHA $120-S 96$ (=S Dor) & $0.173 \pm 0.054$ & $0.172 \pm 0.057$ & $0.374 \pm 0.028$ & $5.586 \pm 0.022$ & LBV \\
\hline LHA 120-S 116 (=R110) & $0.116 \pm 0.034$ & $0.161 \pm 0.036$ & $0.076 \pm 0.032$ & $3.391 \pm 0.034$ & LBV \\
\hline LHA 120-S 128 (=R127) & $0.115 \pm 0.037$ & $0.286 \pm 0.036$ & $0.255 \pm 0.029$ & $6.573 \pm 0.021$ & LBV \\
\hline LHA $120-S 155$ (=R71) & $0.027 \pm 0.035$ & $0.097 \pm 0.038$ & $0.069 \pm 0.033$ & $7.095 \pm 0.024$ & LBV \\
\hline CPD-69 463 (=R143) & $0.244 \pm 0.033$ & $0.201 \pm 0.035$ & $0.259 \pm 0.030$ & $7.851 \pm 0.049$ & LBV \\
\hline LHA $120-S 83(=S k-69142 a)$ & $0.115 \pm 0.041$ & $0.287 \pm 0.041$ & $0.239 \pm 0.031$ & $1.609 \pm 0.055$ & LBV \\
\hline \multicolumn{6}{|c|}{ Small Magellanic Cloud } \\
\hline LHA 115-S 6 & $0.433 \pm 0.034$ & $0.709 \pm 0.0319$ & $0.769 \pm 0.031$ & $4.037 \pm 0.035$ & $\mathrm{~B}[\mathrm{e}] \mathrm{SG}$ \\
\hline LHA $115-S 18$ & $0.418 \pm 0.050$ & $0.822 \pm 0.0460$ & $1.059 \pm 0.030$ & $3.705 \pm 0.031$ & $\mathrm{~B}[\mathrm{e}] \mathrm{SG}$ \\
\hline LHA $115-S 23$ & $0.221 \pm 0.038$ & $0.601 \pm 0.0382$ & $0.946 \pm 0.031$ & $2.484 \pm 0.096$ & $\mathrm{~B}[\mathrm{e}] \mathrm{SG}$ \\
\hline LHA 115-S 65 & $0.292 \pm 0.035$ & $0.800 \pm 0.0311$ & $0.982 \pm 0.030$ & $4.363 \pm 0.027$ & $\mathrm{~B}[\mathrm{e}] \mathrm{SG}$ \\
\hline LHA 115-S 52 (=R40) & $0.182 \pm 0.036$ & $0.065 \pm 0.0330$ & $0.066 \pm 0.031$ & $2.851 \pm 0.043$ & LBV \\
\hline
\end{tabular}

Note: IR photometry for all objects is taken from the 2MASS point source catalog (J, H, and K [104]), except for the stars LHA 120-S 111 [21] and LHA 120-S 89 [108], and from the WISE All-Sky Data Release (W1, W2, and W4 [105]). ${ }^{a}$ Despite of the lack of WISE colors, the presence of warm dust is proven by its IR excess seen in the Spitzer data, and its IR spectrum that looks like a twin of the one of LHA 120-S 73 (see [82]).

\section{A Census of B[e]Sgs}

With clearly defined classification characteristics and the proper observational tools at hand, the massive star population within the local Universe can be scanned for suitable B[e]SG candidates. At the moment of writing this review, this is still an ongoing project that requires patience and sufficient telescope time at both optical and infrared facilities. Nevertheless, many new, particularly extragalactic B[e]SG star discoveries were reported in the literature within the past 20 years. The aim of this section is, therefore, to take a closer and critical look at the suggested B[e]SG candidates in order to sort out possible misclassified objects, to check what type of observations are still missing for unambiguous classification of the candidates, and to compile updated lists of confirmed B[e]SGs for the galaxies with a reported B[e]SG population. The starting points for this investigation are the Magellanic Clouds (Section 4.1), moving further out into the Local Group (Section 4.2) and beyond (Section 4.3), before we finally return to the Milky Way (Section 4.4).

8 One exception to this rule is the LBV star HRCar, which occasionally showed CO band emission related to phases when the star was dimmer [107]. 
The samples in each galaxy are presented in tables, which follow the same structure. The objects are listed under a homogeneous SIMBAD identifier (if possible) in Column 1, reference(s) for the B[e]SG classification of the stars follow in Column 2. Where available, $\mathrm{E}(\mathrm{B}-\mathrm{V})$ values and their references are provide in Columns 3 and 4, and the four colors (J-H, H-K, W1-W2, and W2-W4) are given along with their errors in the last four columns. The tables are furthermore organized such that in the top part appear the confirmed B[e]SGs. These are stars that fulfill all classification criteria. In the middle part of each table stars with uncertain or controversial classification are listed with their names in parentheses. These are objects that lack one or more of the classification criteria due to incomplete observational datasets. Objects for which different research teams find controversial results such that clarification is needed are also included here. In the bottom of each table, erroneously classified objects are gathered with their names in italic and within parentheses. These are stars for which observational evidence (e.g., a specific color) excludes them from belonging to the class of B[e]SGs.

\subsection{Magellanic Clouds}

As mentioned in Section 2, the "classical" sample of B[e]SGs resides within the Magellanic Clouds. The first such object, for which the hybrid character was reported, was the LMC star RMC 126 (LHA 120-S 127 [15]), and soon after followed the identification of ten more B[e]SGs in the LMC and four in the SMC $([16,20,22-24,109]$, see Table 1$)$ based on a dedicated search for similar objects. Since then, a few more stars have been suggested as B[e]SGs candidates. These are presented and discussed in the following. The classical sample of B[e]SGs in the MCs, which fulfill all classification criteria, is listed in Table 1 above, with their IR colors provided in Table 3.

\subsubsection{Large Magellanic Cloud}

New B[e]SG candidates have been found either by dedicated searches, e.g., from cross-matching catalogs of emission-line stars with near-IR catalogs (e.g., [110]), or more serendipitously as a by-product of deep spectroscopic surveys of specific regions, such as the VLT-FLAMES Tarantula Survey (VFTS [111-113]) that was devoted to the 30 Doradus starburst region. In addition, surveys for other purposes, such as the search for post-asymptotic giant branch stars [114], resulted in new B[e]SG candidates ${ }^{9}$. Seven new B[e]SG objects have been proposed in total, of which only two fulfill the criteria for B[e]SGs, two are considered candidates, and three appear to be misclassified. All seven stars are listed in Table 4. Their locations in the two color-color diagrams are shown in Figure 6 in comparison with the confirmed B[e]SGs and LBVs from Table 3.

A sample of confirmed MC late-type stars and supergiants [115] is included in these color-color diagrams. These serve as reference for objects that might have a late-type companion. The outliers of the late-type stars, especially in the WISE diagram, are objects with high mass-loss.

A sample of Galactic Herbig AeBe (HAeBe) stars is also shown [116]. Only stars with known extinction values and solid magnitudes in all four bands were selected (rejecting objects with reported contamination). As some of these pre-main sequence objects suffer from very high extinction $\left(A_{\mathrm{V}}>5 \mathrm{mag}\right.$ ), their colors were corrected using an $R_{\mathrm{V}}$-dependent extinction law [117] with $R_{\mathrm{V}}=5.0$, which has been found to be reasonable for HAeBe stars [116]. These pre-main sequence stars were included to check for possible misclassification of objects that have a proposed luminosity ranging around the lower limit for $\mathrm{B}[\mathrm{e}] \mathrm{SG}$, because this luminosity range is shared by the most massive HAeBes. In the near-IR diagram, the HAeBes populate a stripe that appears to be parallel and seems to connect seamlessly to the region occupied by the classical B[e]SGs. In the WISE diagram, the HAeBes also seem to populate a stripe adjacent to the B[e]SG domain.

9 I would like to point out that from the proposed 12 newly discovered B[e] stars only one was found to be a supergiant (see Table 4). The others have been carefully inspected in collaboration with Devika Kamath, and they did not fulfill the requirements. These results are yet unpublished. 
In the following, the reasons for classification of the new LMC objects as either confirmed or candidate, or for rejecting them as B[e]SGs are briefly presented.

Table 4. Confirmed and candidate B[e]SGs in the LMC. Misclassified objects are listed in the bottom part of the table.

\begin{tabular}{lccccccc}
\hline Object & Ref. & E(B-V) & Ref. & J-H & H-K & W1-W2 & W2-W4 \\
\hline \multicolumn{7}{c}{ Confirmed B[e]SGs } \\
\hline LHA 120-S 165 & {$[114]$} & $\ldots$ & $\ldots$ & $0.735 \pm 0.041$ & $0.98 \pm 0.040$ & $0.886 \pm 0.029$ & $3.385 \pm 0.037$ \\
ARDB 54 & {$[54,118]$} & 0.11 & {$[54]$} & $0.340 \pm 0.057$ & $0.810 \pm 0.04$ & $0.402 \pm 0.023$ & $2.057 \pm 0.054$ \\
\hline \multicolumn{7}{c}{ Uncertain or controversial classification } \\
\hline (VFTS 1003) & {$[111]$} & $\ldots$ & $\ldots$ & $0.540 \pm 0.094$ & $0.87 \pm 0.0539$ & $\ldots$ & $\ldots$ \\
(VFTS 822) & {$[112,113]$} & 0.56 & {$[113]$} & $0.689 \pm 0.039$ & $1.261 \pm 0.035$ & $0.952 \pm 0.033$ & $6.09 \pm 0.046$ \\
\hline \multicolumn{7}{c}{ Erroneous classification } \\
\hline $\begin{array}{l}\text { (VFTS 698) } \\
\text { ([L72] LH 85-10) }\end{array}$ & {$[119]$} & 0.6 & {$[119]$} & $0.436 \pm 0.030$ & $0.444 \pm 0.034$ & $0.585 \pm 0.030$ & $9.81 \pm 0.023$ \\
(NOMAD1 & {$[96]$} & $\ldots$ & $\ldots$ & $0.108 \pm 0.036$ & $0.211 \pm 0.048$ & $\ldots$ & $\ldots$ \\
0181-0125572) & {$[118]$} & $\ldots$ & $\ldots$ & $\ldots{ }^{a}$ & $\ldots{ }^{a}$ & $\ldots$ & $\ldots$ \\
\hline
\end{tabular}

Note: IR photometry for all objects is taken from the 2MASS point source catalog $(\mathrm{J}, \mathrm{H}$, and K [104]) and from the WISE All-Sky Data Release (W1, W2, and W4 [105]). ${ }^{\text {a }}$ The JHK magnitudes listed in SIMBAD were mistakenly taken from the paper of Levato et al. [118], but these belong to the star LHA 120-S 165.

\section{LHA 120-S 165}

This object was first listed as a candidate young stellar object [120], but was later classified as possible B[e]SG, based on its optical spectrum displaying all characteristic emission features (SSTISAGEMC J052747.62-714852.8 [114]). Its optical and infrared brightness together with its position in both color-color diagrams support this classification.

\section{ARDB 54}

Recent analysis of ARDB 54 revealed that it belongs to the (thus far only few) A[e]SGs, being the first of its kind in the LMC [54]. Its position in the near-IR diagram supports this classification, although the object is displaced from the region of classical B[e]SGs in the WISE diagram, where it is located closer to the LBV region. Its luminosity of $\log L / L_{\odot} \simeq 4.4$ [54] is too low to be considered as LBV candidate, and a bit too high to be considered as HAeBe star. From the latter, ARDB 54 is also offset in both color-color diagrams, so that a classification as A[e]SG seems to be the most reasonable, despite its exposed location in the WISE diagram, whose cause should be examined more closely.

\section{(VFTS 1003)}

This star was found from the VLT-FLAMES Tarantula Survey [111]. In high angular-resolution near-IR images, it appears as a single, isolated object [121]. The star's blue emission-line spectrum resembles closely the one of the Galactic B[e]SG GG Car. VFTS 1003 was suggested to be either a Herbig B[e] star or a B[e] supergiant [111]. From its position in the near-IR diagram, a supergiant classification seems more likely. However, the lack of WISE photometry allows assigning VFTS 1003 only a candidate status. In addition, for a definite B[e] classification, red optical spectra are needed, because the optical spectra reported in the literature do not cover the region of the [O I] lines.

\section{(VFTS 822)}

This late B-type star [113] is another object that was identified in the VLT-FLAMES Tarantula Survey [112] based on its blue emission-line spectrum. As with VFTS 1003, a red spectrum is needed to check for the presence of [O I] emission. Similar to that star, VFTS 822 has been proposed as possible 
Herbig B[e] pre-main sequence candidate. It displays a UV excess typical for pre-main sequence stars, but its luminosity of $\log L / L \odot \sim 4$ is not conclusive and leaves as well room for an interpretation as evolved star. In the near-IR diagram, VFTS 822 appears within the B[e]SG domain with a clear separation from the region hosting HAeBe stars, but from the WISE diagram the star is located at the border region with the HAeBes.
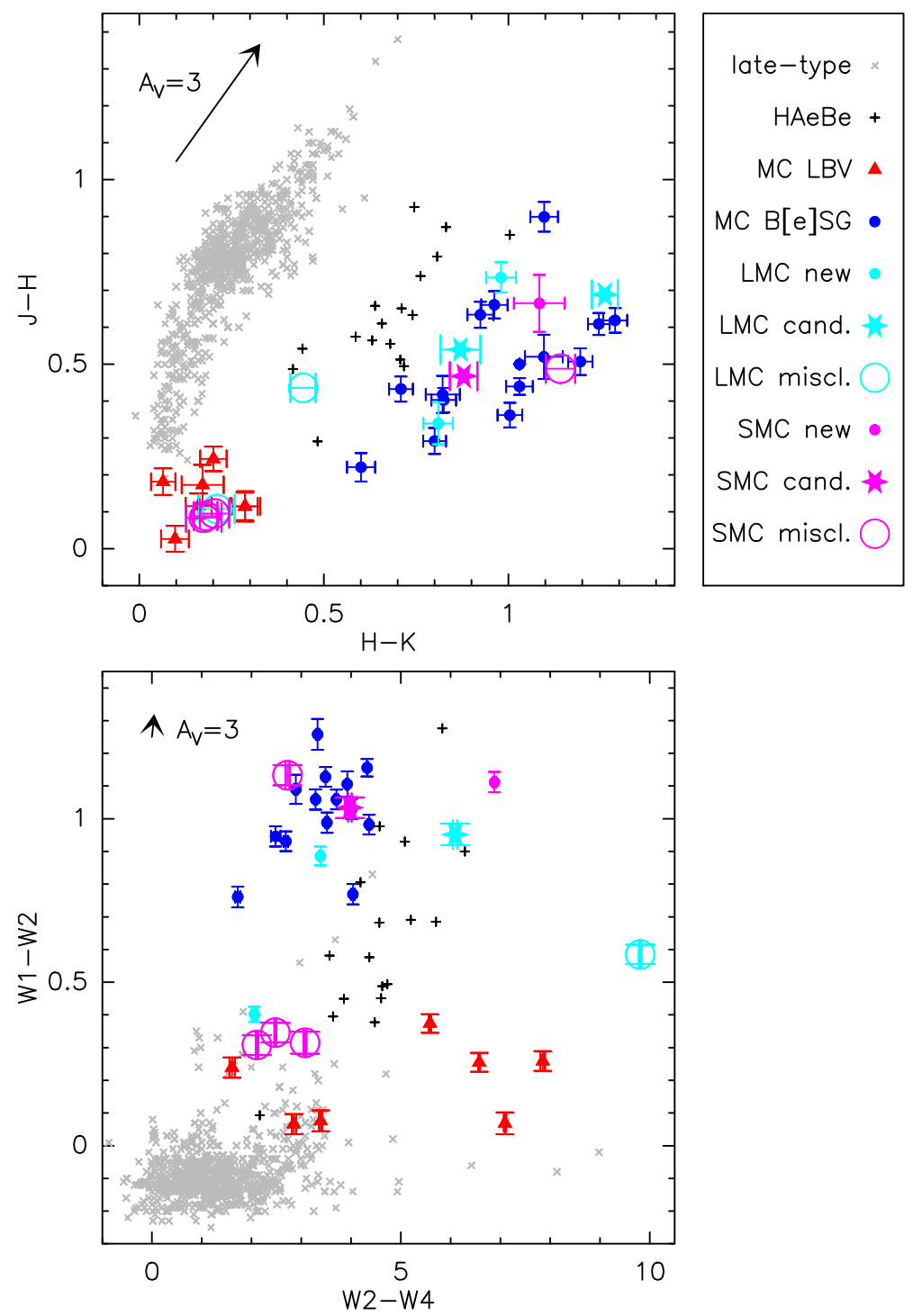

Figure 6. Location of the new LMC (light blue) and SMC (purple) samples with respect to those MC $\mathrm{B}[\mathrm{e}] \mathrm{SG}$ s that meet all the required classification criteria (dark blue) and LBVs (red triangles) in the near-IR (top) and the WISE diagram (bottom). Filled circles are used for confirmed B[e]SGs, filled stars for candidates, and empty circles for misclassified objects. A sample of late-type stars and supergiants in the MCs (small gray crosses, from [115]) and a sample of (dereddened, see text) Galactic HAeBe stars (black plus signs, from [116]) are also included. The arrow in each panel indicates the direction of the reddening, and their length complies with a value of $A_{\mathrm{V}}=3$. As the color excess is not known for all MC objects, no extinction correction has been applied. However, the MC stars have in general relatively small color excess values (see Tables 4 and 5), which would shift them only marginally in the diagrams.

\section{(VFTS 698)}

This is the third object identified within in the VLT-FLAMES Tarantula Survey and suggested as $\mathrm{B}[\mathrm{e}] \mathrm{SG}$ [119]. It also lacks a red optical spectrum to ascertain the presence of [O I] emission in order to 
be considered a $\mathrm{B}[\mathrm{e}]$ star. Its spectral variability and photometric light curve unveiled that the object is most likely an interacting binary consisting possibly of an early B-type star and a veiled more massive companion. Its IR colors place it in both diagrams into the region occupied by HAeBe stars, but the luminosity estimates for both stars with $\log L / L \odot \sim 5$ and 5.3 are too high for a pre-main sequence classification. Correcting for the extinction towards VFTS 698 would shift the object closer to the LBV domain. In the WISE diagram, the object appears particularly far off from the location of the classical $\mathrm{B}[\mathrm{e}] \mathrm{SG}$. Hence, this object needs further investigations for a proper classification.

\section{([L72] LH 85-10)}

Searching for LBV candidates in OB associations in the LMC, the object number 10, within the association LH 85, was found to be a luminous emission-line star. Due to the similarity of its blue spectrum with other B[e]SGs, it has been suggested as a new member of that group [96]. To date, no information about the presence of [O I] emission from that object is available. Moreover, its spectral energy distribution does not display the characteristic near-IR excess emission of B[e]SGs questioning the validity of this classification [108]. The star's position in the near-IR diagram coincides with the region populated by LBVs, supporting the erroneous classification. No WISE photometry is available for this object.

\section{(NOMAD1 0181-0125572)}

This star appears neither in the 2MASS nor in the WISE catalog, although it was reported to have 2MASS photometry [118]. From the coordinates of the source labeled NOMAD1 0181-0125572 by Miroshnichenko et al. [110] it is obvious that it was confused with the object LHA 120-S165. The star with SIMBAD identifier NOMAD1 0181-0125572 is not a B[e]SG.

\subsubsection{Small Magellanic Cloud}

In the SMC, a total of six new B[e]SGs has been reported. Five of them were discovered as by-products from the spectroscopic survey of the hot, luminous stars in the SMC (2dFS [122]), from the Runaways and Isolated O Star Spectroscopic Survey of the SMC (RIOTS4 [123]), and from the SMC photometric catalog [124]. One more object was serendipitously detected [125]. From a critical inspection of the properties of these six stars, only one object appears to fulfill all required criteria, one object appears as very promising candidate that lacks only some complementary information for unambiguous classification, whereas the remaining four stars are considered as misclassified. All objects are presented in the following, their IR colors are provided in Table 5, and their positions within the two color-color diagrams are included in Figure 6.

\section{[MA93] 1116 = NGC 346:KWBBe 200}

The star is one of the few objects residing in a cluster, the SMC cluster NGC 346. Due to its $\mathrm{H} \alpha$ emission, it was first classified as a compact $\mathrm{H}$ II region [126], then as a Be star based on its optical photometry [127], and in the following as B[e]SG based on its optical appearance and near-IR excess emission [125]. In the mid-IR, [MA93] 1116 displays silicate emission and strong PAH bands, features often seen in HAeBe stars (e.g., [128,129]). Additional cold dust is surrounding the object as is implied by its detection at $24 \mu \mathrm{m}$ with Spitzer/MIPS. These characteristics led to the suggestion that [MA93] 1116 might be an evolved young stellar object [130] or a HAeBe star [131], despite the lack of clear evidence for infall, e.g., in form of inverse P Cygni profiles. If true, its luminosity of $\log L / L_{\odot} \sim 4.4[54,125]$ would place it at the upper limit for Herbig objects, while it would get in lane with the group of less-luminous B[e]SGs in the evolved scenario. The position of [MA93] 1116 within the WISE diagram leaves room for a possible classification as HAeBe star where it falls on the edge of the region populated by HAeBe stars, whereas it appears clearly off the HAeBe region in the near-IR color-color diagram. It is hence considered as B[e]SG, and the fifth object within a cluster (the first one in the SMC). 
Table 5. Confirmed and candidate B[e]SGs in the SMC. Misclassified objects are listed in the bottom part of the table.

\begin{tabular}{lccccccc}
\hline Object & Ref. & E(B-V) & Ref. & J-H & H-K & W1-W2 & W2-W4 \\
\hline \multicolumn{7}{c}{ Confirmed B[e]SGs } \\
\hline [MA93] 1116 & {$[54,125]$} & 0.42 & {$[54]$} & $0.665 \pm 0.077$ & $1.084 \pm 0.0689$ & $1.112 \pm 0.03$ & $6.883 \pm 0.034$ \\
\hline \multicolumn{7}{c}{ Uncertain or controversial classification } \\
\hline (LHA 115-S 38) & {$[124]$} & 0.13 & {$[132]$} & $0.468 \pm 0.038$ & $0.879 \pm 0.038$ & $1.034 \pm 0.031$ & $3.978 \pm 0.035$ \\
\hline \multicolumn{7}{c}{ Erroneous classification } \\
\hline (LHA 115-N82) & {$[122,133]$} & 0.12 & {$[133]$} & $0.488 \pm 0.040$ & $1.141 \pm 0.040$ & $1.133 \pm 0.031$ & $2.723 \pm 0.038$ \\
$($ LHA 115-S 29) & {$[123]$} & 0.05 & {$[123]$} & $0.089 \pm 0.030$ & $0.180 \pm 0.032$ & $0.346 \pm 0.030$ & $2.479 \pm 0.020$ \\
(LHA 115-S 46) & {$[123]$} & 0.05 & {$[123]$} & $0.096 \pm 0.037$ & $0.204 \pm 0.040$ & $0.308 \pm 0.030$ & $2.108 \pm 0.020$ \\
$($ LHA 115-S62) & {$[123]$} & 0.11 & {$[123]$} & $0.084 \pm 0.039$ & $0.175 \pm 0.048$ & $0.315 \pm 0.034$ & $3.076 \pm 0.024$ \\
\hline
\end{tabular}

Note: IR photometry for all objects is taken from the 2MASS point source catalog $(\mathrm{J}, \mathrm{H}$, and K [104]) and from the WISE All-Sky Data Release (W1, W2, and W4 [105]).

\section{$($ LHA 115-S $38=2 \mathrm{dFS} 1804)$}

This object was found from the spectroscopic survey of the hot, luminous stars in the SMC [122]. The blue spectrum displays numerous emission lines, in particular of [Fe II], but no information about [OI] emission is available. The spectral energy distribution indicates a near-IR excess [124]. With a luminosity estimate of $\log L / L_{\odot} \simeq 4.1$ the star was also considered as a post-AGB object [132], but its positions within the two color-color diagrams places the star clearly within the B[e]SG domain. As such, it is a strong candidate for another low-luminosity B[e]SG.

$$
(\text { LHA 115-N82 = 2dFS } 2837=\text { LIN 495) }
$$

This star displays all spectroscopic B[e]SG characteristics [122,133] and an IR excess emission [124]. The optical spectrum was reported to be composite [122], and the detected photospheric lines display radial velocity variations, whereas the emission from the circumstellar matter appears to be stable [54]. The IR colors of LHA 115-N82 locate it clearly within the B[e]SG domains. Curiously, the $V$ and $I$ band light curves display a long-term brightening, which resembles LBV outbursts and is not common in B[e]SGs. On the other hand, it displays both [O I] and [Ca II] emission, which are typically not seen in LBVs. Since the star has too low luminosity $\left(\log L / L_{\odot} \simeq 3.8\right)$ to be an LBV (or even a B[e]SG), it was recently assigned a classification as "LBV imposter" [54]. This object clearly requires further investigations to pin down its status.

$$
\text { (LHA 115-S } 29 \text { = RMC 15), (LHA 115-S 46 = RMC 38), (LHA 115-S } 62 \text { = RMC 48) }
$$

The blue optical spectra of these three objects show emission-line features similar to B[e]SGs, and the luminosities derived for these objects, ranging from $\log L / L_{\odot} \simeq 4.4$ to 4.8 , assigns them a supergiant status [123]. However, it is currently not known whether these objects display [O I] line emission, one of the defining characters of the $\mathrm{B}[\mathrm{e}]$ phenomenon. Due to the lack of a pronounced near-IR excess emission, these three stars have been proposed to be dust-poor B[e]SGs [123]. However, the presence of warm dust is another main classification criteria for a star to be considered as B[e]SG. It is not surprising that all three objects fall clearly outside the B[e]SG domains in the color-color diagrams. Instead, their positions coincide with the regions populated by LBVs, which implies that these three stars have dense winds and circumstellar ionized gas, which is exemplified by their emission-line spectra. These objects require further investigations to unveil their true nature.

\subsection{Local Group Galaxies beyond the Magellanic Clouds}

Moving further away, beyond the Magellanic Clouds, we may expect to find B[e]SG stars and candidates in those galaxies, in which star-formation is ongoing. For these galaxies, surveys such as the 
LGGS project mentioned in Section 3, but also earlier surveys (e.g., [134,135]) provide indispensable information on the population of luminous, evolved massive stars, and provide the base for systematic investigations for unambiguous classification of these objects.

To date, systematic spectroscopic studies have been performed in the two large spiral galaxies of the Local Group, M31 and M33, in which a number of B[e]SGs were found amongst the putative LBV candidates $[97,99,136-138]$. The suggested B[e]SG populations in each of these galaxies is presented in the following subsections. The samples are provided in Tables 6 and 7, in which all objects are listed under their Local Group Galaxy Survey (LGGS) identifiers (Column 1). The properties, based on which the decision to categorize an individual star as either a candidate or a misclassified object has been made, are briefly depicted. The locations of the confirmed, candidate, and possibly misclassified $\mathrm{B}[\mathrm{e}] \mathrm{SGs}$ in the two color-color diagrams are shown in Figure 7.

Table 6. Confirmed and candidate B[e]SGs in M31. Misclassified objects are listed at the bottom of the table.

\begin{tabular}{|c|c|c|c|c|c|}
\hline LGGS & Ref. & $\mathbf{J}-\mathbf{H}$ & $\mathbf{H}-\mathbf{K}$ & W1-W2 & W2-W4 \\
\hline \multicolumn{6}{|c|}{ Confirmed B[e]SGs } \\
\hline J004320.97+414039.6 & {$[99,139]$} & 0.358 & $1.522 \pm 0.143$ & $0.983 \pm 0.051$ & $5.284 \pm 0.084$ \\
\hline $\mathrm{J} 004415.00+420156.2^{\mathrm{a}}$ & {$[99,139]$} & $0.741 \pm 0.253$ & $1.510 \pm$ & $0.961 \pm 0.033$ & $3.645 \pm 0.230$ \\
\hline $\mathrm{J} 004417.10+411928.0^{\mathrm{b}, \mathrm{c}}$ & {$[99,136]$} & $0.387 \pm 0.148$ & $0.853 \pm 0.149$ & $0.882 \pm 0.068$ & $4.455 \pm 0.247$ \\
\hline J004522.58+415034. $8^{\mathrm{c}, \mathrm{d}}$ & [136] & $0.684 \pm 0.223$ & $0.877 \pm 0.273$ & $1.091 \pm 0.039$ & $4.303 \pm 0.187$ \\
\hline \multicolumn{6}{|c|}{ Uncertain or controversial classifications } \\
\hline (J004220.31+405123.2) & [99] & .. & & $0.880 \pm 0.062$ & $4.495 \pm 0.298$ \\
\hline (J004221.78+410013.4) & [99] & $\ldots$ & . & $0.927 \pm 0.072$ & $5.253 \pm 0.107$ \\
\hline$(\mathrm{J} 004229.87+410551.8)^{\mathrm{e}}$ & {$[99,139]$} & & & $0.870 \pm 0.045$ & $4.484 \pm 0.082$ \\
\hline (J004411.36+413257.2) & [139] & $0.187 \pm 0.123$ & $0.914 \pm 0.165$ & & \\
\hline (J004442.28+415823.1) & {$[99,139]$} & $\ldots$ & $\ldots$ & $0.781 \pm 0.076$ & $4.994 \pm 0.406$ \\
\hline$(\mathrm{J} 004444.52+412804.0)^{\mathrm{c}, \mathrm{d}}$ & {$[137]$} & $0.576 \pm 0.119$ & $0.858 \pm 0.114$ & $0.883 \pm 0.039$ & $5.247 \pm 0.046$ \\
\hline$(\mathrm{J} 004621.08+421308.2)^{\mathrm{d}}$ & [139] & $0.374 \pm 0.171$ & $1.283 \pm 0.128$ & $1.091 \pm 0.039$ & $4.303 \pm 0.188$ \\
\hline \multicolumn{6}{|c|}{ Erroneous classifications } \\
\hline$(J 004043.10+410846.0)$ & {$[99,139]$} & 1.616 & $0.523 \pm 0.162$ & $0.974 \pm 0.045$ & $4.030 \pm 0.250$ \\
\hline$(J 004057.03+405238.6)$ & [99] & $\ldots$ & $\ldots$ & $\ldots$ & $\ldots$ \\
\hline
\end{tabular}

Note: IR photometry is taken from the 2MASS point source catalog (J, H, K [104]) and from the WISE All-Sky Data Release (W1, W2, W4 [105]). Colors resulting from uncertain photometric values are written in italic. a Possible contamination in the photometric bands J, W1 and W2 due to crowding. ${ }^{b}$ Possible contamination in the photometric bands $\mathrm{J}$ and $\mathrm{H}$ due to crowding. ${ }^{\mathrm{c}} \mathrm{Has}$ CO band emission [136,137]. d Has also been classified as warm hypergiant [140]. e Possible contamination in the photometric bands W1 and W2 due to crowding.

\subsubsection{M31}

A total of $13 \mathrm{~B}[\mathrm{e}] \mathrm{SG}$ s has been proposed in M31 to date. Twelve of them resulted from optical spectroscopic observations of LBV candidates that display the typical B[e]SG characteristics $[99,139]$. Infrared spectroscopic observations of a small LBV candidate sample revealed so far that three display CO band emission [136,137], making their classification as B[e]SG very likely, especially since two of the $\mathrm{CO}$ band emission objects were also found from optical spectroscopy to be a possible B[e]SG. However, a closer look at this total sample of 13 objects, including the available information from nearand mid-IR photometric observations, reveals that only four stars can be considered as confirmed B[e]SGs. Seven objects require further clarifications, and two appear to be misclassified (see Table 6). 
Table 7. Confirmed and candidate B[e]SGs in M33. Misclassified objects are listed at the bottom of the table.

\begin{tabular}{|c|c|c|c|c|c|}
\hline LGGS & Ref. & $\mathbf{J}-\mathbf{H}$ & $\mathbf{H}-\mathbf{K}$ & W1-W2 & W2-W4 \\
\hline \multicolumn{6}{|c|}{ Confirmed B[e]SGs } \\
\hline $\mathrm{J} 013333.22+303343.4^{\mathrm{a}}$ & {$[97,99,138]$} & $1.144 \pm 0.234$ & $1.128 \pm 0.154$ & $0.900 \pm 0.063$ & $5.269 \pm 0.098$ \\
\hline J013350.12+304126.6 & {$[97,99]$} & $0.653 \pm 0.089$ & $0.683 \pm 0.091$ & $0.764 \pm 0.041$ & $4.132 \pm 0.186$ \\
\hline \multicolumn{6}{|c|}{ Uncertain or controversial classifications } \\
\hline (J013324.62+302328.4) & {$[97,99]$} & ... & $\ldots$ & $1.050 \pm 0.047$ & $4.235 \pm 0.414$ \\
\hline (J013342.78+303256.3) & [99] & $\ldots$ & $\ldots$ & $\ldots$ & $\ldots$ \\
\hline (J013349.28+305250.2) & [99] & $\ldots$ & $\ldots$ & $0.746 \pm 0.054$ & $5.394 \pm 0.040$ \\
\hline (J013426.11+303424.7) & {$[97,99]$} & $\ldots$ & $\ldots$ & $1.208 \pm 0.066$ & $4.845 \pm 0.278$ \\
\hline (J013459.47+303701.9) & {$[97,99]$} & $\ldots$ & $\ldots$ & $0.898 \pm 0.053$ & $4.996 \pm 0.038$ \\
\hline (J013500.30+304150.9) & {$[97,99]$} & $\cdots$ & $\ldots$ & $0.939 \pm 0.040$ & $4.142 \pm 0.029$ \\
\hline \multicolumn{6}{|c|}{ Erroneous classifications } \\
\hline$(J 013242.26+302114.1)$ & {$[97,99]$} & $0.861 \pm 0.059$ & $0.376 \pm 0.064$ & $0.050 \pm 0.040$ & $4.543 \pm 0.031$ \\
\hline$(J 013406.63+304147.8)^{b}$ & {$[97,138,141]$} & $0.217 \pm 0.115$ & $0.249 \pm 0.149$ & $\ldots$ & $\ldots$ \\
\hline
\end{tabular}

Note: IR photometry is taken from the 2MASS point source catalog (J, H, and K [104]) and from the WISE All-Sky Data Release (W1, W2, and W4 [105]). Colors resulting from uncertain photometric values are written in italic. ${ }^{\mathrm{a}}$ Has CO band absorption [138]. ${ }^{\mathrm{b}}$ Has CO band emission [138].

\section{J004320.97+414039.6, J004415.00+420156.2, J004417.10+411928.0}

These three objects fulfill all classification criteria of the confirmed B[e]SGs. Their positions in the near-IR and WISE diagrams coincide with the domain populated by the B[e]SGs, except for J004320.97+414039.6, which appears a bit off due to the uncertainties in its $J$ and $H$ magnitudes, which are only upper limits.

\section{J004522.58+415034.8}

The star was assigned a warm hypergiant status due to detected photospheric features pointing towards an A2Ia spectral-type [140]. As it otherwise fulfills all criteria of a B[e]SG (even displaying CO band emission [136]), it is included into the list of confirmed B[e]SGs, but keeping in mind that with its A spectral type it is actually a representative of the (though few) A[e]SGs. Recent modeling of the star's spectral energy distribution resulted in an effective temperature estimate of 11,000 K [142], which would point towards a late-B spectral type.

(J004220.31+405123.2), (J004221.78+410013.4), (J004229.87+410551.8), (J004442.28+415823.1)

These stars display the optical characteristics of B[e]SGs [99], and their WISE colors place them within the B[e]SG domain. However, all four objects lack JHK band photometry, so that they cannot be located in the J-H versus H-K diagram. The presence of He I emission in J004221.78+410013.4 [99] contradicts the proposed effective temperature of $7200 \mathrm{~K}$ [142] and requires clarification.

$$
\text { (J004411.36+413257.2) }
$$

The star is reported to display He I, Fe II, and [Fe II] emission and was suggested as B[e]SG star [139]. Whether it displays [O I] emission was not discussed by these authors and is hence yet unclear, although the spectrum covers that spectral region. Its J-H color is smaller than for typical $\mathrm{B}[\mathrm{e}] \mathrm{SG}$ (but the $\mathrm{H}$ magnitude is uncertain), placing the star at the lower boundary for the B[e]SGs in the color-color diagram. No WISE photometry exists. The closest IR source is more than 6 arcsec away. The object is not reported in other published spectroscopic investigations to display all B[e]SG characteristics. Instead, it was listed as Fe II emission line star [98] and more recently as LBV candidate [142]. 

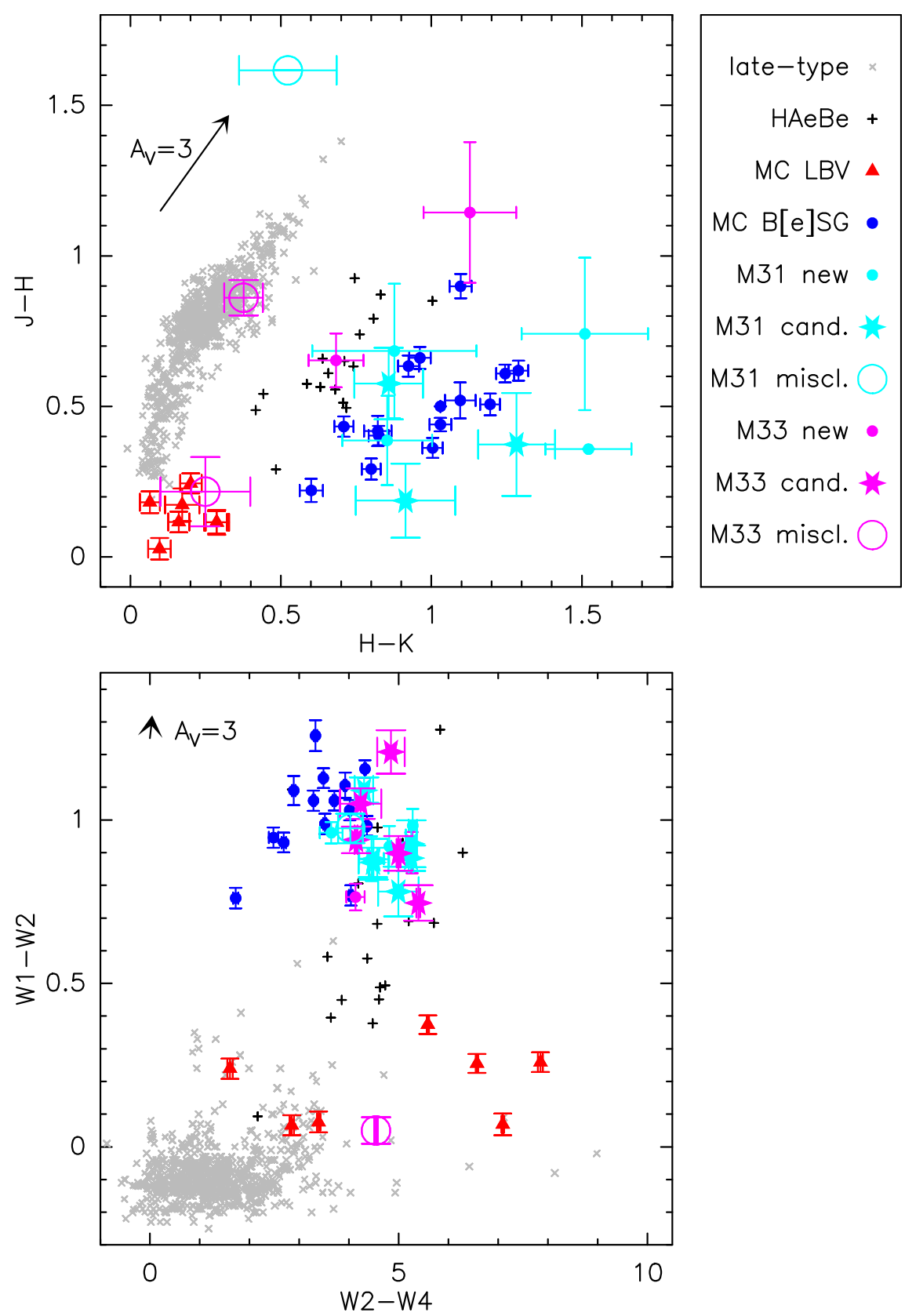

Figure 7. As Figure 6 but for the location of the M31 (light blue) and M33 (purple) samples in the near-IR (top) and the WISE diagram (bottom) based on their observed colors. Typical values for the objects' reddening are $A_{\mathrm{V}} \leq 1.5 \mathrm{mag}$ [142].

(J004444.52+412804.0)

This object was first proposed to be a P Cyg-type LBV candidate [95]. With the detection of [Ca II] emission and circumstellar dust [98], and with the determination of an effective temperature of $6600 \mathrm{~K}$ [142], it was classified as a warm hypergiant. On the other hand, a B[e]SG classification with a central star of 15,000-20,000 K has been suggested, based on the presence of He I lines displaying P Cyg profiles and the detection of intense CO band emission [137]. This controversial classification, based on spectra taken at similar epochs, requires clarification. Infrared photometry places J004444.52+412804.0 within the region occupied by B[e]SGs in the two color-color diagrams. 
(J004621.08+421308.2)

A status of a late A-type warm hypergiant has been assigned to this object based on the lack of detected He I emission [143]. In contrast to this, He I emission lines were reported by Sholukhova et al. [139], and an effective temperature of 10,000 $\mathrm{K}$ has been estimated from the modeling of the stars' spectral energy distribution [142]. The position of the object in the near-IR and WISE diagrams coincides with the B[e]SG domain.

$(J 004043.10+410846.0)$

Its optical spectrum displays all typical characteristics of a B[e]SG [99], and its WISE colors confirm the presence of warm dust, but its (though uncertain) unusually high $\mathrm{J}-\mathrm{H}$ value of 1.6 at a low value of $\mathrm{H}-\mathrm{K}$ fits neither to B[e]SGs nor to LBVs.

$(J 004057.03+405238.6)$

No IR photometry is available, so that currently no statement can be made about the presence of (hot) dust. The absence of He I lines in the optical spectra along with an effective temperature of $7700 \mathrm{~K}$ [142] speak against a B[e]SG classification.

\subsubsection{M33}

In M33, the sample of putative LBV candidates [6,95] served also as a starting point for a more detailed investigation of the individual objects. Optical [97,99,141], and infrared [138] spectroscopic studies revealed so far a total of ten possible B[e]SGs. They are listed in Table 7. Combined with the available information about their infrared colors, only two objects can currently be considered as confirmed B[e]SGs, six need further clarification, and two objects appear to be misclassified.

J013333.22+303343.4, J013350.12+304126.6

Both objects display all classification characteristics of B[e]SGs. Their locations in the WISE diagram coincide with the B[e]SG domain. In the near-IR diagram they appear slightly off the B[e]SG region, shifted towards the HAeBe domain, but their high-luminosities clearly classify them as supergiants. The CO band absorption detected from J013333.22+303343.4 cannot be explained with a cool companion, but might originate from a pole-on seen jet [138]. Whether also CO band emission is present in this object, but veiled by the intense absorption component, is currently unknown.

(J013324.62+302328.4), (J013342.78+303256.3), (J013349.28+305250.2), (J013426.11+303424.7), (J013459.47+303701.9), (J013500.30+304150.9)

All six objects lack near-IR photometry, which renders it difficult to unambiguously classify them as B[e]SGs, although their mid-IR colors and their optical spectroscopic appearances support such a classification. The star J013342.78+303256.3 has even no mid-IR photometry, so that it is unclear whether it is surrounded by (warm) dust at all. There is an IR source at a distance of $\sim 4.5$ arcsec which is considered as too far off to be identified with J013342.78+303256.3. The sources J013324.62+302328.4 and J013500.30+304150.9 were reported as intrinsically faint objects with little extinction, and were suggested as counterparts of the low-luminosity B[e]SG in the MCs [97]. However, according to the luminosity estimates for these stars, for which values of $\log L / L_{\odot} \simeq 4.8-5.0$ were obtained [142], these stars fall rather onto the lower boundary of the high-luminosity B[e]SGs sample within the MCs (see Figure 1).

(J013242.26+302114.1)

The optical spectra of this object display all typical B[e]SG characteristics, but the star's IR colors displace it clearly from the sample of confirmed B[e]SGs. It has excess emission at $8 \mu \mathrm{m}$ due to PAHs [99]. The JHK magnitudes suggest that J013242.26+302114.1 falls into the region of late-type 
stars and stars with a late-type companion, respectively, [20,144]. This classification is supported by the WISE colors, although regular late-type stars typically have slightly negative W1-W2 colors. Considering only the WISE photometry, J013242.26+302114.1 would fit to the region populated by LBVs. This objects clearly needs to be investigated in more detail for a proper classification.

\section{$(J 013406.63+304147.8)$}

This star is also known as [HS80] B416. Despite the detected CO band emission from this object [138], a classification as B[e]SG seems not appropriate (as previously mentioned by Humphreys et al. $[98,99]$ ), because its near-IR colors show clearly the lack of warm dust and would place the object to the region populated by LBVs. In addition, no [OI] emission is seen from J013406.63+304147.8 [98]. However, thus far, no photometric variability, typical for LBVs, is recorded [97], and the star can only be considered as a possible LBV candidate, as was previously suggested [99]. The star is found to be surrounded by an expanding ring-like nebula [141] and shows excess emission at $8 \mu \mathrm{m}$ due to PAHs [138]. The spectral energy distribution leaves space for a companion [98,138], and its spectral lines display radial velocity variations with a period of $16.13 \mathrm{~d}$, leading to the suggestion that the system might be an interacting binary system causing mass loss in the equatorial plane [145].

\subsection{Beyond the Local Group}

Not much is known yet about the B[e]SG population beyond the Local Group. Most promising for dedicated searches are the nearby large spiral galaxies M81, M101, and NGC 2403, for which pioneering ground-based surveys have been conducted already in the 1980s and 1990s [146-149] and which are nowadays extended to fainter objects thanks to the capabilities of the Hubble Space Telescope (e.g., [150]). Spectroscopic follow-up investigations revealed a number of variable luminous objects [151-155], but only in one of these galaxies, M81, three B[e]SG candidates have been found so far [156]. They all display the typical B[e] features in their optical spectra, but without complementary information about the presence of (warm) circumstellar dust, their classification remains preliminary. These objects will certainly not remain the only of their kind, because the search for more candidates has just begun.

The B[e]SG candidates in M81 are listed in Table 8. For lack of proper SIMBAD identifiers for these objects, the table contains the star ID [156] along with the coordinates and, where available, the V band magnitudes.

Table 8. Candidate B[e]SGs in M81.

\begin{tabular}{lcccc}
\hline Star ID & RA(J2000) & Dec(J2000) & V & Ref. \\
\hline$(10584-8-4)$ & $9: 54: 50.03$ & $+69: 06: 55.47$ & $\ldots$ & {$[156]$} \\
$(10584-4-1)$ & $9: 54: 54.05$ & $+69: 10: 23.00$ & 19.68 & {$[156]$} \\
$(10584-9-1)$ & $9: 55: 18.97$ & $+69: 08: 27.54$ & 19.10 & {$[156]$} \\
\hline
\end{tabular}

In the past few years, additional surveys have been performed, using large ground-based facilities (e.g., [157]) and space telescopes (e.g., [158,159]). These surveys were aimed at revealing the luminous and variable massive star populations of other galaxies even further away. They provide an excellent basis for follow-up spectroscopic studies to classify their massive star content, so that many more LBV candidates and B[e]SGs may be found in the (near) future. In addition, the Local Group galaxies still need to be explored in more detail, in particular those galaxies that were already found to possess (even though in very small numbers) LBV candidates [95] that are awaiting their proper classifications. The upcoming era of the Extreme Large Telescope (ELT) promises to become particularly fruitful. The next generation of high-sensitivity instruments combined with the large collecting area of the telescope will facilitate ground-based spectroscopic observations of faint objects with very high spatial resolution. 


\subsection{Galactic Objects}

Finally, we return to our own Galaxy. Searching for B[e]SG stars in the Milky Way is a difficult task. Though many $\mathrm{B}[\mathrm{e}]$ stars are known, the assignment of a supergiant status is significantly hampered due to highly uncertain distances, hence luminosities. The situation will hopefully change with the final data release from the GAIA mission, from which one hopes for accurate parallax measurements. However, for now, the luminosities of the objects are subject to large uncertainties, so that only objects with a reported luminosity of at least $\log L / L_{\odot} \geq 5$ are considered as serious B[e]SG candidates.

The lower luminosity boundary of $\log L / L_{\odot} \sim 4.0$ for an evolved star to be assigned a supergiant status is a further hindrance in the classification of objects as B[e]SGs, because this luminosity domain is shared with the massive pre-main sequence (HAeBe) stars. The latter have emission-line spectra with numerous forbidden emission lines from [Fe II] and [O I] similar to the B[e]SGs, and the stars are surrounded by significant amounts of circumstellar dust within their massive accretion disks causing considerable IR excess emission, just as the B[e]SGs. Hence, it is not surprising that confusion exists about the proper classification for a number of objects within this luminosity domain of $4.0<\log L / L_{\odot}<4.5$, and that Galactic B[e]SG candidates also appear as candidates in catalogs of HAeBe stars (see, e.g., [160]). In the absence of clear indications for infall of material, which is a typical characteristic of pre-main sequence stars, alternative discriminators for the classification of such objects are needed.

A reasonable approach to this is to search for ${ }^{13} \mathrm{CO}$ emission from the circumstellar environments of the uncertain candidates. Many HAeBe stars have been reported to display CO band emission from their massive accretion disks (e.g., [161-165]). As these disks form from material provided by the interstellar medium in which the ${ }^{12} \mathrm{C} /{ }^{13} \mathrm{C}$ isotope abundance ratio has typically a value of about 90 [88], these pre-main sequence disks can clearly be distinguished from the disks around evolved massive stars, which should be enriched in ${ }^{13} \mathrm{C}$ and hence give rise to clearly measurable emission in ${ }^{13} \mathrm{CO}$. K-band spectra of these objects, covering the first-overtone bands of both ${ }^{12} \mathrm{CO}$ and ${ }^{13} \mathrm{CO}$ (see Section 2.3 and Figure 3), are thus key for a proper discrimination between a young (pre-main sequence) and an evolved status.

From the currently proposed 15 Galactic B[e]SGs listed in Table 2, nine have been reported to display $\mathrm{CO}$ band emission. However, thus far, only four of them have been observed in the region around the ${ }^{13} \mathrm{CO}$ bands. The spectra of all four stars have been found to display clear signatures of ${ }^{13} \mathrm{CO}$ emission, and model results revealed that the environments of all four objects are clearly enriched in ${ }^{13} \mathrm{C}$. Two of these objects were already known to be supergiants based on their confirmed high luminosities: the stars GG Car [41,42] and Hen 2-398 [41]. For the other two, which so far have also been considered as HAeBe candidates (see Table9), the detection of chemically processed material can hence be regarded as the ultimate proof of their evolved, supergiant nature. These are the objects MWC 137 [59] and MWC 349 [62]. These results are very promising and encouraging, and they demonstrate that the ${ }^{13} \mathrm{CO}$ molecular emission provides a solid tool to unambiguously classify a star as either a pre-main sequence or an evolved object. Clearly, more observational effort needs to be undertaken to search also for the signatures of ${ }^{13} \mathrm{CO}$ in the spectra of the remaining objects.

When collecting the IR magnitudes of the Galactic sample, it turned out that only the near-IR measurements are reliable, whereas the WISE measurements for all objects have been flagged as being contaminated by neighboring objects. The latter are hence useless for classification purposes, and one can currently only rely on the JHK-band magnitudes. The list of objects, their observed colors, and literature values of their color excess are listed in Table 9. The relatively high values of the color excess requires correction for extinction before placing the objects to the near-IR diagram. Corrections have been performed with the galactic extinction curve using an $R_{\mathrm{V}}$ value of 3.1 [117]. The extinction corrected colors are included in Table 9, and the positions of the objects are shown in Figure 8 separately for the confirmed (Figure 8, top) and candidate objects (Figure 8, bottom). 
Table 9. Confirmed and candidate B[e]SGs in the Milky Way.

\begin{tabular}{|c|c|c|c|c|c|c|c|}
\hline Object & Ref. & $E(B-V)$ & Ref. & $(\mathrm{J}-\mathrm{H})$ & $(\mathrm{H}-\mathrm{K})$ & $(\mathrm{J}-\mathrm{H})_{0}$ & $(\mathrm{H}-\mathrm{K})_{0}$ \\
\hline \multicolumn{8}{|c|}{ Confirmed B[e]SGs } \\
\hline MWC $137^{a}$ & {$[59,166]$} & 1.22 & [166] & $0.922 \pm 0.040$ & $1.217 \pm 0.035$ & $0.572 \pm 0.040$ & $0.930 \pm 0.035$ \\
\hline MWC $349^{\text {a }}$ & {$[62,167]$} & $\sim 3.2$ & [168] & $1.472 \pm 0.038$ & $1.603 \pm 0.369$ & $0.554 \pm 0.038$ & $0.851 \pm 0.369$ \\
\hline GGCar b & {$[41,42,169]$} & 0.51 & [169] & $0.818 \pm 0.049$ & $0.964 \pm 0.049$ & $0.672 \pm 0.049$ & $0.844 \pm 0.049$ \\
\hline Hen 3-298 & {$[41,63]$} & 1.7 & [63] & $1.009 \pm 0.065$ & $1.139 \pm 0.060$ & $0.522 \pm 0.065$ & $0.739 \pm 0.060$ \\
\hline CPD-52 9243 & [50] & 1.7 & [35] & $0.919 \pm 0.029$ & $0.948 \pm 0.026$ & $0.432 \pm 0.029$ & $0.548 \pm 0.026$ \\
\hline HD $327083^{b}$ & [170] & 1.8 & [170] & $1.000 \pm 0.042$ & $1.309 \pm 0.188$ & $0.484 \pm 0.042$ & $0.886 \pm 0.188$ \\
\hline MWC $300^{a, b}$ & {$[171,172]$} & 1.2 & [172] & $1.150 \pm 0.056$ & $1.951 \pm 0.055$ & $0.806 \pm 0.056$ & $1.669 \pm 0.055$ \\
\hline AS $381^{b}$ & [65] & 2.3 & [65] & $1.285 \pm 0.029$ & $1.117 \pm 0.028$ & $0.625 \pm 0.029$ & $0.576 \pm 0.028$ \\
\hline CPD-57 2874 & [47] & 1.75 & [47] & $0.803 \pm 0.062$ & $0.678 \pm 0.304$ & $0.301 \pm 0.062$ & $0.267 \pm 0.304$ \\
\hline \multicolumn{8}{|c|}{ Uncertain or controversial classifications } \\
\hline (Hen 3-938) & [54] & 1.64 & [54] & $1.593 \pm 0.035$ & $1.487 \pm 0.034$ & $1.124 \pm 0.035$ & $1.101 \pm 0.034$ \\
\hline$(\mathrm{MWC} 342)^{\mathrm{a}, \mathrm{b}}$ & [173] & 1.5 & [173] & $1.179 \pm 0.027$ & $1.121 \pm 0.025$ & $0.749 \pm 0.027$ & $0.768 \pm 0.025$ \\
\hline (Hen 3-303) & [63] & 1.7 & [63] & $1.366 \pm 0.063$ & $1.443 \pm 0.063$ & $0.879 \pm 0.063$ & $1.042 \pm 0.063$ \\
\hline$(\mathrm{CD}-4211721)^{\mathrm{a}}$ & [67] & $1.4-1.6$ & {$[50,67]$} & $1.317 \pm 0.053$ & $1.398 \pm 0.050$ & $0.901 \pm 0.053$ & $1.057 \pm 0.050$ \\
\hline$(\mathrm{HD} 87643)^{\mathrm{a}, \mathrm{b}}$ & {$[35,174]$} & 1.0 & [35] & $1.461 \pm 0.271$ & $1.300 \pm 0.338$ & $1.174 \pm 0.271$ & $1.065 \pm 0.338$ \\
\hline$(\mathrm{HD} 62623)^{b}$ & [69] & 0.17 & [175] & $0.395 \pm 0.380$ & $0.693 \pm 0.368$ & $0.346 \pm 0.380$ & $0.653 \pm 0.368$ \\
\hline
\end{tabular}

Note: IR photometry is taken from the 2MASS point source catalog (J, H, and K [104]). ${ }^{\text {a }}$ Star appears also in HAeBe catalogs (see, e.g., [160]). ${ }^{\mathrm{b}}$ Confirmed or suspected binary.

The separation of confirmed from candidate B[e]SGs is based on two characteristics: (i) stars with detected enrichment in ${ }^{13} \mathrm{CO}$ of their circumstellar environments are considered as confirmed; and (ii) stars with reported (by more than one research team) luminosity values of $\log L / L_{\odot} \geq 5.0$. Objects with lower luminosities $4.0<\log L / L_{\odot}<5.0$ are assigned a candidate status. Based on these criteria, the Galactic sample splits into nine confirmed B[e]SGs and six candidates (see Table 9).

\section{MWC 137, MWC 349, GG Car, Hen 3-298}

These four objects are considered as confirmed B[e]SGs based on the detected enrichment of their circumstellar disk material with ${ }^{13} \mathrm{CO}$ (see Table 2). They all fall into the region of the confirmed $\mathrm{B}[\mathrm{e}] \mathrm{SGs}$ in the near-IR diagram, regardless of the large error bar for MWC 349.

\section{CPD-52 9243, HD 327083, MWC 300}

All three stars fulfill the high luminosity criterion. The near-IR colors of CPD-52 9243 and HD 327083 place these two objects within the B[e]SG domain. MWC 300 appears regularly in studies of HAeBe stars. Its near-IR colors locate this star close to the B[e]SGs but far away from the HAeBe region, making a pre-main sequence nature of this object rather unlikely. Its relatively high color values might be influenced by a possible companion [51,172].

\section{AS 381, CPD-57 2874}

These two objects are also known to have high luminosities, but they reside slightly outside the classical B[e]SG domain in the near-IR diagram. AS 381 is a reported binary $[64,65]$ consisting of a luminous B[e]SG and a K-type companion, which seems to (significantly) contribute to the total near-IR flux, hence altering the colors of the B[e]SG. The near-IR colors of CPD-57 2874 place it closer to the LBVs rather than to the $\mathrm{B}[\mathrm{e}] \mathrm{SGs}$, although its $\mathrm{H}-\mathrm{K}$ color is subject to large uncertainty. Its pronounced emission in $\mathrm{CO}$, and in the [O I] and [Ca II] forbidden lines [58] speak against an LBV classification.

(Hen 3-938), (MWC 342), (Hen 3-303), (CD-42 11721)

The rather low luminosities of these four objects and the closeness or even coincidence of their location with the HAeBe domain clearly requires further studies for an unambiguous classification. While Hen 3-938 and MWC 342 have to our knowledge not yet been spectroscopically observed in 
the K-band, the other two stars showed no evidence for CO band emission [39]. This renders their classification more difficult.
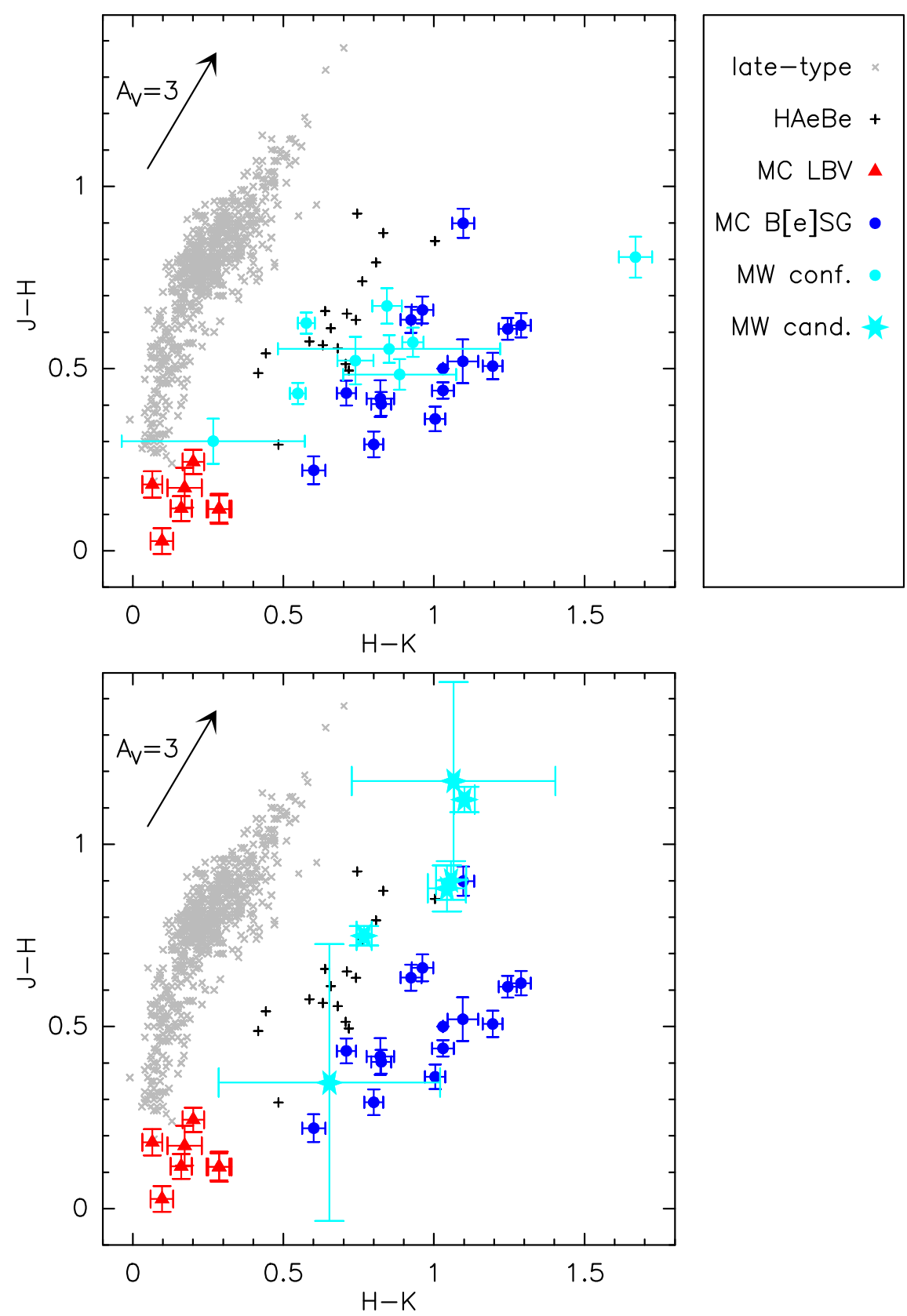

Figure 8. Near-IR diagrams as in Figure 6, showing the locations of the Galactic confirmed B[e]SGs (top) and B[e]SG candidates (bottom). The colors of the Galactic objects have been corrected for interstellar extinction (Table 9).

(HD 87643)

The near-IR colors of HD 87643 place it to the high end of the HAeBe regime. However, these colors have large errors and require refinement. Studies based on long-baseline interferometry revealed that this object consists of two B-type stars, and each component might be surrounded by a dusty disk [174]. Whether this object is a physical binary is currently not known. 
(HD 62623)

It appeared in the literature as the first A[e]SG $[49,176]$ and is known to be surrounded by a detached gas and dust disk, from which also CO band emission has been detected [39,58]. It has been speculated that the gap between the star and the inner rim of the gas disk might have been cleared by a companion. However, no clear evidence of such a companion has been found yet. Its low luminosity and large errors in the near-IR photometric measurements make it challenging to unambiguously assign the star a B[e]SG status, although its position within the near-IR seems to coincide more with the B[e]SG domain rather than with any other classification. This object is an ideal candidate to clarify its nature based on dedicated K-band observations to analyze the ${ }^{13} \mathrm{CO}$ content within its circumstellar disk.

\section{Discussion and Conclusions}

B[e]SGs form a special class of evolved massive stars and are thought to represent a short-lived transition phase either in their red-ward, post-main sequence evolution, or in their blue-ward post-RSG evolution. The total number of currently known B[e]SGs is low, supporting the idea of a short transition phase. However, in which direction the stars evolve, and whether all B[e]SGs evolve in the same direction, is still an open issue. For the objects in M31 and M33, it has been argued that the B[e]SGs are more isolated than LBVs and hardly found in stellar associations, so that a post-RSG (or post-yellow supergiant) evolution was proposed to be more likely [142]. This assessment was based on the available numbers of putative B[e]SGs in these two galaxies at that time. However, after revision of the two samples (Tables 6 and 7), two of the four confirmed B[e]SGs in M31 and both confirmed B[e]SGs in M33 are associated with stellar groups, questioning the conclusion that B[e]SGs are isolated and thus post-RSGs.

The best age indicator for B[e]SGs we have to date is their surface abundance enrichment in ${ }^{13} \mathrm{C}$, as discussed in Section 2.3. If B[e]SGs were post-RSGs, their progenitors would all have started with a (very) low rotation speed. While such a scenario cannot be excluded, it may not be very likely considering that stars are born on average with a rotation rate of about $40 \%$ of their critical velocity [88].

Interaction within a (close) binary system, possibly even up to a binary merger, seems to be an alternative and popular scenario (e.g., [51,177]), but the number of currently confirmed binaries amongst B[e]SGs is still rather low to give preference to the binary channel as the sole possible way for the formation of B[e]SGs. Likewise, some B[e]SGs have been suggested as suitable supernova candidates. For example, the Galactic object MWC 137 appears similar to Sher 25 [178] and SBW1 [179], which both look like the progenitor of SN1987A. In addition, the SMC object LHA 115-S 18 has been proposed to be a viable SN1987A progenitor [27]. In this respect, it is vital to resolve B[e]SG populations and to study their properties.

In this review, a census of the currently known B[e]SG population in the Milky Way and in nearby star-forming galaxies within and beyond the Local Group is presented. The proposed candidates have been undertaken a critical examination, sorted into confirmed B[e]SGs and B[e]SG candidates, and unsuitable objects have been flagged as "misclassified"10.

During these investigations, a fundamental difference has been recognized between the identification issues for objects in our Galaxy compared to those in other galaxies. Extragalactic B[e]SGs bare the risk of being confused with LBVs in quiescence, which share very similar optical spectroscopic characteristics. To separate these two classes of objects, one can make use of clearly defined classification criteria based on certain sets of emission features identified in their optical and near-IR spectra, as outlined in Section 3. In addition, inspection of the location of possible B[e]SG candidates in

10 We would like to caution that, with insufficient knowledge of stellar properties, individual objects may easily be misclassified, as it happened in recently published catalogs $[6,102,180,181]$, in which erroneously a number of (even confirmed) B[e]SGs are listed as LBV candidates. 
the IR color-color diagrams is highly advisable, because B[e]SGs and LBVs populate clearly separate domains. This fact might also be used as starting point for future investigations of extragalactic samples. However, for such future studies, infrared photometry with higher spatial resolution than what is currently provided by 2MASS and WISE is desirable to prevent from contamination with neighboring sources in densely populated regions. Moreover, precise distances, as soon provided by GAIA, will help to separate foreground stars in the directions to other galaxies that might have been misclassified as luminous extragalactic stars.

In the Milky Way, an additional complication occurs due to the often uncertain luminosity estimates, and the overlap of low-luminosity B[e]SGs with the most luminous massive pre-main sequence objects (HAeBe). Here, special care needs to be taken, especially since both classes of objects occupy adjacent regions in the near-IR color-color diagram with a probable overlap. Without additional distinctive features for such low-luminosity, borderline B[e]SG candidates, their real nature remains elusive. One complementary classification criteria that was discussed, is provided by the enrichment of the circumstellar material of evolved objects with processed material that has been released from the stellar surface, as opposed to the non-processed material with interstellar abundance patterns found around HAeBes. The most ideal element to search for is ${ }^{13} \mathrm{C}$, which is bound in ${ }^{13} \mathrm{CO}$ molecules in the circumstellar disks. Measuring the ${ }^{13} \mathrm{CO}$ amount with respect to ${ }^{12} \mathrm{CO}$ provides immediate insight into the nature of the object. The current HAeBe samples might hide such low-luminosity B[e]SG candidates, which can only be identified as such by careful and honest analysis.

The result from this census, after strict application of the classification criteria, is that we count nine confirmed and six candidate B[e]SGs in the Galaxy. Moving out to the MCs, the numbers amount to $13(+2)$ in the LMC and $5(+1)$ in the SMC. The situation in other members of the Local Group is not much better, where the numbers drop to four (+7) in M31 and two (+6) in M33. Even further away, only three candidates have been reported from M81. The total number of B[e]SGs found in the various galaxies are too small for statistical analyses with respect to a metallicity dependence of their number, but the comparable quantities within the Milky Way and the LMC suggest only a mild dependence of the amount of B[e]SGs on (i.e., a possible drop with decreasing) metallicity.

Further, we report that the Galactic sample of 15 B[e]SGs and candidates contains currently seven confirmed or suspected binaries (see Table 9), which is less than half of the population. In the MCs, this number is even lower, because thus far only three stars have been reported to be possible binaries, of which one, the SMC star LHA 115-S6 (= RMC 4) has been proposed to be the remnant of a binary merger within an initially triple system $[182,183]$. The other two objects, the LMC star LHA 120-S 134 and the SMC object LHA115-S18, have been identified as optical counterparts of $\mathrm{X}$-ray sources $[27,29]$. Nothing is known about possible binarity in the B[e]SG samples from the other galaxies, although photometric variability was seen in at least two M31 objects: the confirmed B[e]SG star J004417.10+411928.0 [137,184] and the candidate J004444.52+412804.0 $[185,186]$. Whether this variability is a sign of binarity or just of semi-regular variability which might be interpreted with pulsation activity such as reported from the $\alpha$ Cygni variables, needs to be studied in more detail.

For the sake of completeness, a special class of objects, which have not been discussed yet, should be briefly mentioned as well. These are the high-mass X-ray binaries (HMXB) with possible B[e] supergiant (candidate) companion. Some objects with an assigned B[e]SG status have been found to be too luminous in X-rays for being considered single stars. These objects have been proposed to be binary systems, in which the high energy emission is caused by either accretion onto a compact object, or by shocks in a colliding wind binary with a second massive star. Members of this group are the Galactic objects $\mathrm{Cl}^{*}$ Westerlund $1 \mathrm{~W} 9$ (=Wd1-9), which is considered a colliding wind system [85]; CICam (=MWC 84), which might be interpreted as supernova imposter [187]; and the high-mass X-ray binary (HMXB) IGR J16318-4848, in which the compact object was proposed to be a neutron star [188]. Two ultra-luminous X-ray sources (ULXs), Holmberg II X-1 and NGC 300 ULX1, the latter being also named as supernova imposter SN2010da, have also been proposed to be HMXBs including a B[e]SG [189-191]. Whether these objects indeed host a B[e]SG clearly needs to be investigated in 
more detail. However, since the behavior of these sources is considerably different from the confirmed B[e]SGs, I hesitate to include them into the census.

The presented populations represent the current knowledge of B[e]SGs and B[e]SG candidates in the closest star-forming galaxies. With the ever growing sensitivities of instruments and telescope sizes, the future in B[e]SG star research is bright, because many more candidates will be identified in even more distant galaxies and with metallicities spreading over a large range. With statistically meaningful samples, it will finally be possible to unveil the nature and fate of these fascinating objects.

Funding: This project received funding from the Grant Agency of the Czech Republic (GAČR, grant number 17-02337S). The Astronomical Institute of the Czech Academy of Sciences, Ondřejov, is supported by the project RVO:67985815.

Acknowledgments: I wish to thank the editor, Roberta Humphreys, for the invitation to write this review. In addition, I am grateful to Michalis Kourniotis for inspiring discussions on searching for extragalactic B[e]SGs, and to Dieter Nickeler and Lydia Cidale for passionate discussions about B[e]SGs as well as for their proofreading of and suggestions on the draft versions. Moreover, I thank the anonymous referees for their careful reading and suggestions on the draft version. This research made use of the NASA Astrophysics Data System (ADS) and of the SIMBAD database, operated at CDS, Strasbourg, France. This publication makes use of data products from the 2MASS, which is a joint project of the University of Massachusetts and the Infrared Processing and Analysis Center/California Institute of Technology, funded by the National Aeronautics and Space Administration and the National Science Foundation. This publication makes use of data products from the Wide-field Infrared Survey Explorer, which is a joint project of the University of California, Los Angeles, and the Jet Propulsion Laboratory/California Institute of Technology, funded by the National Aeronautics and Space Administration.

Conflicts of Interest: The author declares no conflict of interest.

\section{References}

1. Groh, J.H.; Georgy, C.; Ekström, S. Progenitors of supernova Ibc: A single Wolf-Rayet star as the possible progenitor of the SN Ib iPTF13bvn. Astron. Astrophys. 2013, 558, L1. [CrossRef]

2. Groh, J.H.; Meynet, G.; Georgy, C.; Ekström, S. Fundamental properties of core-collapse supernova and GRB progenitors: Predicting the look of massive stars before death. Astron. Astrophys. 2013, 558, A131. [CrossRef]

3. Groh, J.H.; Meynet, G.; Ekström, S. Massive star evolution: Luminous blue variables as unexpected supernova progenitors. Astron. Astrophys. 2013, 550, L7. [CrossRef]

4. Pauldrach, A.W.A.; Vanbeveren, D.; Hoffmann, T.L. Radiation-driven winds of hot luminous stars XVI. Expanding atmospheres of massive and very massive stars and the evolution of dense stellar clusters. Astron. Astrophys. 2012, 538, A75. [CrossRef]

5. Hopkins, P.F.; Kereš, D.; Oñorbe, J.; Faucher-Giguère, C.-A.; Quataert, E.; Murray, N.; Bullock, J.S. Galaxies on FIRE (Feedback in Realistic Environments): Stellar feedback explains cosmologically inefficient star formation. Mon. Not. R. Astron. Soc. 2014, 445, 581-603. [CrossRef]

6. Massey, P.; Neugent, K.F.; Smart, B.M. A Spectroscopic Survey of Massive Stars in M31 and M33. Astron. J. 2016, 152, 62. [CrossRef]

7. Cannon, A.J. Peculiar Spectra in the Large Magellanic Cloud. Harv. Coll. Obs. Bull. 1924, 801, 1-3.

8. Henize, K.G. Catalogues of $\mathrm{H} \alpha$-emission Stars and Nebulae in the Magellanic Clouds. Astrophys. J. Suppl. 1956, 2, 315. [CrossRef]

9. Smith, H.J. Spectra of Bright-Line Stars in the Large Magellanic Cloud. Publ. ASP 1957, 69, 137. [CrossRef]

10. Feast, M.W.; Thackeray, A.D.; Wesselink, A.J. The brightest stars in the Magellanic Clouds. Mon. Not. R. Astron. Soc. 1960, 121, 337. [CrossRef]

11. Allen, D.A.; Glass, I.S. Emission-line stars in the Large Magellanic Cloud: Spectroscopy and infrared photometry. Astrophys. J. 1976, 210, 666-669. [CrossRef]

12. Glass, I.S. A dusty star in the Small Magellanic Cloud. Mon. Not. R. Astron. Soc. 1977, 178, 9P. [CrossRef]

13. Stahl, O.; Wolf, B.; Zickgraf, F.J.; Bastian, U.; de Groot, M.H.J.; Leitherer, C. R66 (Aeq), an LMC B Supergiant with a Massive, Cool and Dusty Wind. Astron. Astrophys. 1983, 120, 287-296.

14. Stahl, O.; Leitherer, C.; Wolf, B.; Zickgraf, F.-J. Three new hot stars with dust shells in the Magellanic clouds. Astron. Astrophys. 1984, 131, L5-L6.

15. Zickgraf, F.-J.; Wolf, B.; Stahl, O.; Leitherer, C.; Klare, G. The hybrid spectrum of the LMC hypergiant R 126. Astron. Astrophys. 1985, 143, 421-430. 
16. Zickgraf, F.J.; Wolf, B.; Stahl, O.; Leitherer, C.; Appenzeller, I. B(e)-supergiants of the Magellanic Clouds. Astron. Astrophys. 1986, 163, 119-134.

17. Gray, D.F. The Observation and Analysis of Stellar Photospheres; Cambridge University Press: Cambridge, UK, 2005.

18. Zickgraf, F.-J. B[e] Supergiants in the Magellanic Clouds. In Stars with the B[e] Phenomenon; ASP Conference Series; Astronomical Society of the Pacific: San Francisco, CA, USA, 2006; Volume 355, p. 135.

19. Georgy, C.; Ekström, S.; Eggenberger, P.; Meynet, G.; Haemmerlé, L.; Maeder, A.; Granada, A.; Groh, J.H.; Hirschi, R.; Mowlavi, N.; et al. Grids of stellar models with rotation. III. Models from 0.8 to $120 \mathrm{M}_{\odot}$ at a metallicity $\mathrm{Z}=0.002$. Astron. Astrophys. 2013, 558, A103. [CrossRef]

20. Gummersbach, C.A.; Zickgraf, F.-J.; Wolf, B. B[e] phenomenon extending to lower luminosities in the Magellanic Clouds. Astron. Astrophys. 1995, 302, 409.

21. McGregor, P.J.; Hillier, D.J.; Hyland, A.R. CO Overtone Emission from Magellanic Cloud Supergiants. Astrophys. J. 1988, 334, 639. [CrossRef]

22. Zickgraf, F.-J.; Kovacs, J.; Wolf, B.; Stahl, O.; Kaufer, A.; Appenzeller, I. R4 in the Small Magellanic Cloud: A spectroscopic binary with a B[e]/LBV-type component. Astron. Astrophys. 1996, 309, 505-514.

23. Zickgraf, F.-J.; Wolf, B.; Stahl, O.; Humphreys, R.M. S 18: A new B(e) supergiant in the Small Magellanic Cloud with evidence for an excretion disk. Astron. Astrophys. 1989, 220, 206-214.

24. Zickgraf, F.-J.; Stahl, O.; Wolf, B. IR survey of OB emission-line stars in the SMC: Detection of a new B E supergiant, AV 172. Astron. Astrophys. 1992, 260, 205-212.

25. Lamers, H.J.G.L.M.; Zickgraf, F.-J.; de Winter, D.; Houziaux, L.; Zorec, J. An improved classification of B[e]-type stars. Astron. Astrophys. 1998, 340, 117-128.

26. Appenzeller, I.; Klare, G.; Stahl, O.; Wolf, B.; Zickgraf, F.-J. CASPEC/Cassegrain Echelle Spectrograph/and IUE-A Perfect Match. Messenger 1984, 38, 28.

27. Clark, J.S.; Bartlett, E.S.; Coe, M.J.; Dorda, R.; Haberl, F.; Lamb, J.B.; Negueruela, I.; Udalski, A. The supergiant B[e] star LHA 115-S 18-Binary and/or luminous blue variable? Astron. Astrophys. 2013, 560, A10. [CrossRef]

28. Massey, P.; Neugent, K.F.; Morrell, N.; Hillier, D.J. A Modern Search for Wolf-Rayet Stars in the Magellanic Clouds: First Results. Astrophys. J. 2014, 788, 83. [CrossRef]

29. Bartlett, E.S.; Clark, J.S. Observational Constraints on the Nature of the X-ray bright supergiant B[e] stars LHA 115-S18 \& LHA 120- S134. In Proceedings of the SALT Science Conference, Stellenbosch, South Afrcia, 1-5 June 2015.

30. Magalhaes, A.M. Polarization and the Envelopes of B[e] Supergiants in the Magellanic Clouds. Astrophys. J. 1992, 398, 286. [CrossRef]

31. Melgarejo, R.; Magalhães, A.M.; Carciofi, A.C.; Rodrigues, C.V. S 111 and the polarization of the B[e] supergiants in the Magellanic Clouds. Astron. Astrophys. 2001, 377, 581-588. [CrossRef]

32. Magalhães, A.M.; Melgarejo, R.; Pereyra, A.; Carciofi, A.C. Polarimetry and the Envelopes of Magellanic B[e] Supergiants. In Stars with the B[e] Phenomenon; ASP Conference Series; Astronomical Society of the Pacific: San Francisco, CA, USA, 2006; Volume 355, p. 147.

33. Seriacopi, D.B.; Carciofi, A.C.; Magalhães, A.M. The Envelopes of B[e] Supergiants in the Magellanic Clouds as Seen by Polarimetry. In The B[e] Phenomenon: Forty Years of Studies; Miroshnichenko, A., Zharikov, S., Korčáková, D., Wolf, M., Eds.; ASP Conference Series; Astronomical Society of the Pacific: San Francisco, CA, USA, 2017; Volume 508, p. 109.

34. Zickgraf, F.-J.; Schulte-Ladbeck, R.E. Polarization characteristics of galactic Be stars. Astron. Astrophys. 1989, 214, 274-284.

35. McGregor, P.J.; Hyland, A.R.; Hillier, D.J. Atomic and Molecular Line Emission from Early-Type High-Luminosity Stars. Astrophys. J. 1988, 324, 1071. [CrossRef]

36. McGregor, P.J.; Hyland, A.R.; McGinn, M.T. Emission-line stars in the Magellanic Clouds: Infrared spectroscopy of Be and Ofpe/WN9 stars. Astron. Astrophys. 1989, 223, 237-240.

37. Morris, P.W.; Eenens, P.R.J.; Hanson, M.M.; Conti, P.S.; Blum, R.D. Infrared Spectra of Massive Stars in Transition: WNL, Of, Of/WN, Be, B[e], and Luminous Blue Variable Stars. Astrophys. J. 1996, 470, 597. [CrossRef]

38. Wheelwright, H.E.; de Wit, W.J.; Weigelt, G.; Oudmaijer, R.D.; Ilee, J.D. AMBER and CRIRES observations of the binary sgB[e] star HD 327083: Evidence of a gaseous disc traced by CO bandhead emission. Astron. Astrophys. 2012, 543, A77. [CrossRef] 
39. Muratore, M.F.; Kraus, M.; de Wit, W.J. Near-infrared spectroscopic survey of galactic B[e] stars. Boletín Asoc. Argent. Astron. 2012, 55, 123-127.

40. Oksala, M.E.; Kraus, M.; Arias, M.L.; Borges Fernandes, M.; Cidale, L.; Muratore, M.F.; Curé, M. The sudden appearance of CO emission in LHA 115-S 65. Mon. Not. R. Astron. Soc. 2012, 426, L56-L60. [CrossRef]

41. Oksala, M.E.; Kraus, M.; Cidale, L.S.; Muratore, M.F.; Borges Fernandes, M. Probing the ejecta of evolved massive stars in transition. A VLT/SINFONI K-band survey. Astron. Astrophys. 2013, 558, A17. [CrossRef]

42. Kraus, M.; Oksala, M.E.; Nickeler, D.H.; Muratore, M.F.; Borges Fernandes, M.; Aret, A.; Cidale, L.S.; de Wit, W.J. Molecular emission from GG Carinae's circumbinary disk. Astron. Astrophys. 2013, 549, A28. [CrossRef]

43. Kraus, M.; Cidale, L.S.; Arias, M.L.; Maravelias, G.; Nickeler, D.H.; Torres, A.F.; Borges Fernandes, M.; Aret, A.; Curé, M.; Vallverdú, R.; et al. Inhomogeneous molecular ring around the B[e] supergiant LHA 120-S 73. Astron. Astrophys. 2016, 593, A112. [CrossRef]

44. Torres, A.F.; Cidale, L.S.; Kraus, M.; Arias, M.L.; Barbá, R.H.; Maravelias, G.; Borges Fernandes, M. Resolving the clumpy circumstellar environment of the B[e] supergiant LHA 120-S 35. Astron. Astrophys. 2018, 612, A113. [CrossRef]

45. Kraus, M.; Oksala, M.E.; Cidale, L.S.; Arias, M.L.; Torres, A.F.; Borges Fernandes, M. Discovery of SiO Band Emission from Galactic B[e] Supergiants. Astrophys. J. Lett. 2015, 800, L20. [CrossRef]

46. Torres, A.F.; Kraus, M.; Cidale, L.S.; Barba, R.; Borges Fernandes, M.; Brandi, E. Discovery of Raman-scattered lines in the massive luminous emission-line star LHA 115-S 18. Mon. Not. R. Astron. Soc. 2012, 427, L80-L84. [CrossRef]

47. Domiciano de Souza, A.; Driebe, T.; Chesneau, O.; Hofmann, K.-H.; Kraus, S.; Miroshnichenko, A.S.; Ohnaka, K.; Petrov, R.G.; Preisbisch, T.; Stee, P.; et al. AMBER/VLTI and MIDI/VLTI spectro-interferometric observations of the $\mathrm{B}[\mathrm{e}]$ supergiant $\mathrm{CPD}-57^{\mathrm{deg}} 2874$. Size and geometry of the circumstellar envelope in the near- and mid-IR. Astron. Astrophys. 2007, 464, 81-86. [CrossRef]

48. Domiciano de Souza, A.; Bendjoya, P.; Niccolini, G.; Chesneau, O.; Borges Fernandes, M.; Carciofi, A.C.; Spang, A.; Stee, P.; Driebe, T. Fast ray-tracing algorithm for circumstellar structures (FRACS). II. Disc parameters of the B[e] supergiant CPD-57 ${ }^{\mathrm{deg}} 2874$ from VLTI/MIDI data. Astron. Astrophys. 2011, 525, A22. [CrossRef]

49. Millour, F.; Meilland, A.; Chesneau, O.; Stee, P.; Kanaan, S.; Petrov, R.; Mourard, D.; Kraus, S. Imaging the spinning gas and dust in the disc around the supergiant A[e] star HD 62623. Astron. Astrophys. 2011, 526, A107. [CrossRef]

50. Cidale, L.S.; Borges Fernandes, M.; Andruchow, I.; Arias, M.L.; Kraus, M.; Chesneau, O.; Kanaan, S.; Curé, M.; de Wit, W.J.; Muratore, M.F. Observational constraints for the circumstellar disk of the B[e] star CPD-52 9243. Astron. Astrophys. 2012, 548, A72. [CrossRef]

51. Wang, Y.; Weigelt, G.; Kreplin, A.; Hofmann, K.-H.; Kraus, S.; Miroshnichenko, A.S.; Schertl, D.; Chelli, A.; Domiciano de Souza, A.; Massi, F.; et al. AMBER/VLTI observations of the B[e] star MWC 300. Astron. Astrophys. 2012, 545, L10. [CrossRef]

52. Wheelwright, H.E.; de Wit, W.J.; Oudmaijer, R.D.; Vink, J.S. VLTI/AMBER observations of the binary B[e] supergiant HD 327083. Astron. Astrophys. 2012, 538, A6. [CrossRef]

53. Aret, A.; Kraus, M.; Muratore, M.F.; Borges Fernandes, M. A new observational tracer for high-density disc-like structures around B[e] supergiants. Mon. Not. R. Astron. Soc. 2012, 423, 284-293. [CrossRef]

54. Condori, C.A.H.; Borges Fernandes, M.; Kraus, M.; Panoglou, D.; Guerrero, C.A. The study of unclassified B[e] stars and candidates in the Galaxy and Magellanic Clouds. Mon. Not. R. Astron. Soc. 2019, 488, 1090-1110. [CrossRef]

55. Kraus, M.; Liimets, T.; Cappa, C.E.; Cidale, L.S.; Nickeler, D.H.; Duronea, N.U.; Arias, M.L.; Gunawan, D.S.; Oksala, M.E.; Borges Fernandes, M.; et al. Resolving the Circumstellar Environment of the Galactic B[e] Supergiant Star MWC 137 from Large to Small Scales. Astron. J. 2017, 154, 186. [CrossRef]

56. Kraus, M.; Borges Fernandes, M.; de Araújo, F.X. On the hydrogen neutral outflowing disks of B[e] supergiants. Astron. Astrophys. 2007, 463, 627-634. [CrossRef]

57. Kraus, M.; Borges Fernandes, M.; de Araújo, F.X. Neutral material around the B[e] supergiant star LHA 115-S 65. An outflowing disk or a detached Keplerian rotating disk? Astron. Astrophys. 2010, 517, A30. [CrossRef] 
58. Maravelias, G.; Kraus, M.; Cidale, L.S.; Borges Fernandes, M.; Arias, M.L.; Curé, M.; Vasilopoulos, G. Resolving the kinematics of the discs around Galactic B[e] supergiants. Mon. Not. R. Astron. Soc. 2018, 480, 320-344. [CrossRef]

59. Muratore, M.F.; Kraus, M.; Oksala, M.E.; Arias, M.L.; Cidale, L.; Borges Fernandes, M.; Liermann, A. Evidence of the Evolved Nature of the B[e] Star MWC 137. Astron. J. 2015, 149, 13. [CrossRef]

60. Aret, A.; Kraus, M.; Šlechta, M. Spectroscopic survey of emission-line stars-I. B[e] stars. Mon. Not. R. Astron. Soc. 2016, 456, 1424-1437. [CrossRef]

61. Kraus, M.; Krügel, E.; Thum, C.; Geballe, T.R. CO band emission from MWC 349. I. First overtone bands from a disk or from a wind? Astron. Astrophys. 2000, 362, 158-168.

62. Kraus, M.; Arias, M.L.; Cidale, L.S.; Torres, A.F. Evidence of an evolved nature of MWC 349A. 2019, in preparation.

63. Miroshnichenko, A.S.; Bjorkman, K.S.; Grosso, M.; Hinkle, K.; Levato, H.; Marang, F. Properties of galactic B[e] supergiants. IV. Hen 3-298 and Hen 3-303. Astron. Astrophys. 2005, 436, 653-659. [CrossRef]

64. Liermann, A.; Schnurr, O.; Kraus, M.; Kreplin, A.; Arias, M.L.; Cidale, L.S. A K-band spectral mini-survey of Galactic B[e] stars. Mon. Not. R. Astron. Soc. 2014, 443, 947-956. [CrossRef]

65. Miroshnichenko, A.S.; Bjorkman, K.S.; Chentsov, E.L.; Klochkova, V.G.; Ezhkova, O.V.; Gray, R.O.; García-Lario, P.; Perea Calderón, J.V.; Rudy, R.J.; Lynch, D.K.; et al. The luminous B[e] binary AS 381. Astron. Astrophys. 2002, 383, 171-181. [CrossRef]

66. Andrillat, Y.; Jaschek, C. B[e] stars. VIII. MWC 342. Astron. Astrophys. Suppl. 1999, 136, 59-63. [CrossRef]

67. Borges Fernandes, M.; Kraus, M.; Lorenz Martins, S.; de Araújo, F.X. On the evolutionary stage of the unclassified B[e] star CD-42 ${ }^{\circ} 11721$. Mon. Not. R. Astron. Soc. 2007, 377, 1343-1362. [CrossRef]

68. Kraus, M.; Borges Fernandes, M.; Kubát, J.; de Araújo, F.X. From B[e] to A[e]. On the peculiar variations of the SMC supergiant LHA 115-S 23 (AzV 172). Astron. Astrophys. 2008, 487, 697-707. [CrossRef]

69. Chentsov, E.L.; Klochkova, V.G.; Miroshnichenko, A.S. Spectral variability of the peculiar A-type supergiant 3Pup. Astrophys. Bull. 2010, 65, 150-163. [CrossRef]

70. Kraus, M.; Lamers, H.J.G.L.M. Ionization structure in the winds of B[e] supergiants. I. Ionization equilibrium calculations in a H plus He wind. Astron. Astrophys. 2003, 405, 165-174. [CrossRef]

71. Kraus, M. Ionization structure in the winds of B[e] supergiants. II. Influence of rotation on the formation of equatorial hydrogen neutral zones. Astron. Astrophys. 2006, 456, 151-159. [CrossRef]

72. Zsargó, J.; Hillier, D.J.; Georgiev, L.N. Axi-symmetric models of B[e] supergiants. I. The effective temperature and mass-loss dependence of the hydrogen and helium ionization structure. Astron. Astrophys. 2008, 478, 543-551. [CrossRef]

73. Glatzel, W.; Kiriakidis, M. Stability of Massive Stars and the Humphreys-Davidson Limit. Mon. Not. R. Astron. Soc. 1993, 263, 375-384. [CrossRef]

74. Kiriakidis, M.; Fricke, K.J.; Glatzel, W. The stability of massive stars and its dependence on metallicity and opacity. Mon. Not. R. Astron. Soc. 1993, 264, 50-62. [CrossRef]

75. Glatzel, W.; Mehren, S. Non-radial pulsations and stability of massive stars. Mon. Not. R. Astron. Soc. 1996, 282, 1470-1482. [CrossRef]

76. Kurfürst, P.; Feldmeier, A.; Krtička, J. Time-dependent modeling of extended thin decretion disks of critically rotating stars. Astron. Astrophys. 2014, 569, A23. [CrossRef]

77. Kurfürst, P.; Feldmeier, A.; Krtička, J. Two-dimensional modeling of density and thermal structure of dense circumstellar outflowing disks. Astron. Astrophys. 2018, 613, A75. [CrossRef]

78. Pelupessy, I.; Lamers, H.J.G.L.M.; Vink, J.S. The radiation driven winds of rotating B[e] supergiants. Astron. Astrophys. 2000, 359, 695-706.

79. Curé, M. The Influence of Rotation in Radiation-driven Wind from Hot Stars: New Solutions and Disk Formation in Be Stars. Astrophys. J. 2004, 614, 929-941. [CrossRef]

80. Curé, M.; Rial, D.F.; Cidale, L. Outflowing disk formation in B[e] supergiants due to rotation and bi-stability in radiation driven winds. Astron. Astrophys. 2005, 437, 929-933. [CrossRef]

81. Kraus, M. Spectroscopic Diagnostics for Circumstellar Disks of B[e] Supergiants. In The B[e] Phenomenon: Forty Years of Studies; Miroshnichenko, A., Zharikov, S., Korčáková, D., Wolf, M., Eds.; ASP Conference Series; Astronomical Society of the Pacific: San Francisco, CA, USA, 2017; Volume 508, pp. 219-228.

82. Kastner, J.H.; Buchanan, C.; Sahai, R.; Forrest, W.J.; Sargent, B.A. The Dusty Circumstellar Disks of B[e] Supergiants in the Magellanic Clouds. Astron. J. 2010, 139, 1993-2002. [CrossRef] 
83. Mehner, A.; de Wit, W.J.; Groh, J.H.; Oudmaijer, R.D.; Baade, D.; Rivinius, T.; Selman, F.; Boffin, H.M.J.; Martayan, C. VLT/MUSE discovers a jet from the evolved B[e] star MWC 137. Astron. Astrophys. 2016, 585, A81. [CrossRef]

84. Clark, J.S.; Negueruela, I.; Crowther, P.A.; Goodwin, S.P. On the massive stellar population of the super star cluster Westerlund 1. Astron. Astrophys. 2005, 434, 949-969. [CrossRef]

85. Clark, J.S.; Ritchie, B.W.; Negueruela, I. The circumstellar environment and evolutionary state of the supergiant B[e] star Wd1-9. Astron. Astrophys. 2013, 560, A11. [CrossRef]

86. Liermann, A.; Kraus, M.; Schnurr, O.; Borges Fernandes, M. The ${ }^{13}$ Carbon footprint of B[e] supergiants. Mon. Not. R. Astron. Soc. 2010, 408, L6-L10. [CrossRef]

87. Kraus, M. The pre- versus post-main sequence evolutionary phase of $\mathrm{B}[\mathrm{e}]$ stars. Constraints from $13 \mathrm{CO}$ band emission. Astron. Astrophys. 2009, 494, 253-262. [CrossRef]

88. Ekström, S.; Georgy, C.; Eggenberger, P.; Meynet, G.; Mowlavi, N.; Wyttenbach, A.; Granada, A.; Decressin, T.; Hirschi, R.; Frischknecht, U.; et al. Grids of stellar models with rotation. I. Models from 0.8 to $120 M_{\odot}$ at solar metallicity $(\mathrm{Z}=0.014)$. Astron. Astrophys. 2012, 537, A146. [CrossRef]

89. Berkhuijsen, E.M.; Humphreys, R.M.; Ghigo, F.D.; Zumach, W. A catalogue of the brightest stars in the field of M 31. Astron. Astrophys. Suppl. 1988, 76, 65-99.

90. Massey, P.; Armandroff, T.E.; Conti, P.S. Massive stars in M31. Astron. J. 1986, 92, 1303-1333. [CrossRef]

91. Freedman, W.L. The Young Stellar Content of Nearby Resolved Galaxies. Ph.D. Thesis, University of Toronto, Toronto, ON, Canada, 1984.

92. Humphreys, R.M.; Massey, P.; Freedman, W.L. Spectroscopy of Luminous Blue Stars in M31 and M33. Astron. J. 1990, 99, 84. [CrossRef]

93. Massey, P.; Olsen, K.A.G.; Hodge, P.W.; Strong, S.B.; Jacoby, G.H.; Schlingman, W.; Smith, R.C. A Survey of Local Group Galaxies Currently Forming Stars. I. UBVRI Photometry of Stars in M31 and M33. Astron. J. 2006, 131, 2478-2496. [CrossRef]

94. Massey, P.; Olsen, K.A.G.; Hodge, P.W.; Jacoby, G.H.; McNeill, R.T.; Smith, R.C.; Strong, S.B. A Survey of Local Group Galaxies Currently Forming Stars. II. UBVRI Photometry of Stars in Seven Dwarfs and a Comparison of the Entire Sample. Astron. J. 2007, 133, 2393-2417. [CrossRef]

95. Massey, P.; McNeill, R.T.; Olsen, K.A.G.; Hodge, P.W.; Blaha, C.; Jacoby, G.H.; Smith, R.C.; Strong, S.B. A Survey of Local Group Galaxies Currently Forming Stars. III. A Search for Luminous Blue Variables and Other H $\alpha$ Emission-Line Stars. Astron. J. 2007, 134, 2474-2503. [CrossRef]

96. Massey, P.; Waterhouse, E.; DeGioia-Eastwood, K. The Progenitor Masses of Wolf-Rayet Stars and Luminous Blue Variables Determined from Cluster Turnoffs. I. Results from 19 OB Associations in the Magellanic Clouds. Astron. J. 2000, 119, 2214-2241. [CrossRef]

97. Clark, J.S.; Castro, N.; Garcia, M.; Herrero, A.; Najarro, F.; Negueruela, I.; Ritchie, B.W.; Smith, K.T. On the nature of candidate luminous blue variables in M 33. Astron. Astrophys. 2012, 541, A146. [CrossRef]

98. Humphreys, R.M.; Weis, K.; Davidson, K.; Bomans, D.J.; Burggraf, B. Luminous and Variable Stars in M31 and M33. II. Luminous Blue Variables, Candidate LBVs, Fe II Emission Line Stars, and Other Supergiants. Astrophys. J. 2014, 790, 48. [CrossRef]

99. Humphreys, R.M.; Gordon, M.S.; Martin, J.C.; Weis, K.; Hahn, D. Luminous and Variable Stars in M31 and M33. IV. Luminous Blue Variables, Candidate LBVs, B[e] Supergiants, and the Warm Hypergiants: How to Tell Them Apart. Astrophys. J. 2017, 836, 64. [CrossRef]

100. Gvaramadze, V.V.; Kniazev, A.Y.; Fabrika, S. Revealing evolved massive stars with Spitzer. Mon. Not. R. Astron. Soc. 2010, 405, 1047-1060. [CrossRef]

101. Wachter, S.; Mauerhan, J.C.; Van Dyk, S.D.; Hoard, D.W.; Kafka, S.; Morris, P.W. A Hidden Population of Massive Stars with Circumstellar Shells Discovered with the Spitzer Space Telescope. Astron. J. 2010, 139, 2330-2346. [CrossRef]

102. Aadland, E.; Massey, P.; Neugent, K.F.; Drout, M.R. Shedding Light on the Isolation of Luminous Blue Variables. Astron. J. 2018, 156, 294. [CrossRef]

103. Campagnolo, J.C.N.; Borges Fernandes, M.; Drake, N.A.; Kraus, M.; Guerrero, C.A.; Pereira, C.B. Detection of new eruptions in the Magellanic Clouds luminous blue variables R 40 and R 110. Astron. Astrophys. 2018, 613, A33. [CrossRef] 
104. Cutri, R.M.; Skrutskie, M.F.; van Dyk, S.; Beichman, C.A.; Carpenter, J.M.; Chester, T.; Cambresy, L.; Evans, T.; Fowler, J.; Gizis, J.; et al. VizieR Online Data Catalog: 2MASS All-Sky Catalog of Point Sources. VizieR On-line Data Catalog 2003, II/246.

105. Cutri, R.M. VizieR Online Data Catalog: WISE All-Sky Data Release. VizieR On-line Data Catalog 2012, II/311.

106. Worthey, G.; Lee, H. An Empirical UBV RI JHK Color-Temperature Calibration for Stars. Astrophys. J. Suppl. 2011, 193, 1. [CrossRef]

107. Morris, P.W.; Voors, R.H.M.; Lamers, H.J.G.L.M.; Eenens, P.R.J. Near-Infrared Spectra of LBV; Be and B[e] Stars: Does Axisymmetry Provide a Morphological Link? In Luminous Blue Variables: Massive Stars in Transition; Nota, A., Lamers, H., Eds.; ASP Conference Series; Astronomical Society of the Pacific: San Francisco, CA, USA, 1997; Volume 120, p. 20.

108. Bonanos, A.Z.; Massa, D.; Sewilo, M.; Lennon, D.J.; Panagia, N.; Smith, L.J.; Meixner, M.; Babler, B.L.; Bracker, S.; Meade, M.R.; et al. Spitzer SAGE Infrared Photometry of Massive Stars in the Large Magellanic Cloud. Astron. J. 2009, 138, 1003-1021. [CrossRef]

109. Stahl, O.; Smolinski, J.; Wolf, B.; Zickgraf, F.-J. High-Dispersion Spectroscopy of the B[e] Supergiant S 111. In Physics of Luminous Blue Variables; Davidson, K., Moffat, A.F.J., Lamers, H.J.G.L.M., Eds.; Astrophysics and Space Science Library: Dordrecht, The Netherlands; Kluwer Academic Publishers: Boston, MA, USA, 1989; Volume 157, p. 295.

110. Miroshnichenko, A.S.; Manset, N.; Polcaro, F.; Rossi, C.; Zharikov, S. The B[e] phenomenon in the Milky Way and Magellanic Clouds. Proc. Int. Astron. Union 2011, 6, 260-264. [CrossRef]

111. Evans, C.J.; Taylor, W.D.; Hénault-Brunet, V.; Sana, H.; de Koter, A.; Simón-Díaz, S.; Carraro, G.; Bagnoli, T.; Bastian, N.; Bestenlehner, J.M.; et al. The VLT-FLAMES Tarantula Survey. I. Introduction and observational overview. Astron. Astrophys. 2011, 530, A108. [CrossRef]

112. Evans, C.J.; Kennedy, M.B.; Dufton, P.L.; Howarth, I.D.; Walborn, N.R.; Markova, N.; Clark, J.S.; de Mink, S.E.; de Koter, A.; Dunstall, P.R.; et al. The VLT-FLAMES Tarantula Survey. XVIII. Classifications and radial velocities of the B-type stars. Astron. Astrophys. 2015, 574, A13. [CrossRef]

113. Kalari, V.M.; Vink, J.S.; Dufton, P.L.; Evans, C.J.; Dunstall, P.R.; Sana, H.; Clark, J.S.; Ellerbroek, L.; de Koter, A.; Lennon, D.J.; et al. The VLT-FLAMES Tarantula Survey. XV. VFTS 822: A candidate Herbig B[e] star at low metallicity. Astron. Astrophys. 2014, 564, L7. [CrossRef]

114. Kamath, D.; Wood, P.R.; Van Winckel, H. Optically visible post-AGB stars, post-RGB stars and young stellar objects in the Large Magellanic Cloud. Mon. Not. R. Astron. Soc. 2015, 454, 1468-1502. [CrossRef]

115. González-Fernández, C.; Dorda, R.; Negueruela, I.; Marco, A. A new survey of cool supergiants in the Magellanic Clouds. Astron. Astrophys. 2015, 578, A3. [CrossRef]

116. Hernández, J.; Calvet, N.; Briceño, C.; Hartmann, L.; Berlind, P. Spectral Analysis and Classification of Herbig Ae/Be Stars. Astron. J. 2004, 127, 1682-1701. [CrossRef]

117. Cardelli, J.A.; Clayton, G.C.; Mathis, J.S. The Relationship between Infrared, Optical, and Ultraviolet Extinction. Astrophys. J. 1989, 345, 245. [CrossRef]

118. Levato, H.; Miroshnichenko, A.S.; Saffe, C. New objects with the B[e] phenomenon in the Large Magellanic Cloud. Astron. Astrophys. 2014, 568, A28. [CrossRef]

119. Dunstall, P.R.; Fraser, M.; Clark, J.S.; Crowther, P.A.; Dufton, P.L.; Evans, C.J.; Lennon, D.J.; Soszyński, I.; Taylor, W.D.; Vink, J.S. The VLT-FLAMES Tarantula Survey. V. The peculiar B[e]-like supergiant, VFTS698, in 30 Doradus. Astron. Astrophys. 2012, 542, A50. [CrossRef]

120. Whitney, B.A.; Sewilo, M.; Indebetouw, R.; Robitaille, T.P.; Meixner, M.; Gordon, K.; Meade, M.R.; Babler, B.L.; Harris, J.; Hora, J.L.; et al. Spitzer Sage Survey of the Large Magellanic Cloud. III. Star Formation and 1000 New Candidate Young Stellar Objects. Astron. J. 2008, 136, 18-43. [CrossRef]

121. Campbell, M.A.; Evans, C.J.; Mackey, A.D.; Gieles, M.; Alves, J.; Ascenso, J.; Bastian, N.; Longmore, A.J. VLT-MAD observations of the core of 30 Doradus. Mon. Not. R. Astron. Soc. 2010, 405, 421-435. [CrossRef]

122. Evans, C.J.; Howarth, I.D.; Irwin, M.J.; Burnley, A.W.; Harries, T.J. A 2dF survey of the Small Magellanic Cloud. Mon. Not. R. Astron. Soc. 2004, 353, 601-623. [CrossRef]

123. Graus, A.S.; Lamb, J.B.; Oey, M.S. Discovery of New, Dust-poor B[e] Supergiants in the Small Magellanic Cloud. Astrophys. J. 2012, 759, 10. [CrossRef]

124. Bonanos, A.Z.; Lennon, D.J.; Köhlinger, F.; van Loon, J.T.; Massa, D.L.; Sewilo, M.; Evans, C.J.; Panagia, N.; Babler, B.L.; Block, M.; et al. Spitzer SAGE-SMC Infrared Photometry of Massive Stars in the Small Magellanic Cloud. Astron. J. 2010, 140, 416-429. [CrossRef] 
125. Wisniewski, J.P.; Bjorkman, K.S.; Bjorkman, J.E.; Clampin, M. Discovery of a New Dusty B[e] Star in the Small Magellanic Cloud. Astrophys. J. 2007, 670, 1331-1336. [CrossRef]

126. Meyssonnier, N.; Azzopardi, M. A new catalogue of $\mathrm{H}$-alpha emission-line stars and small nebulae in the Small Magellanic Cloud. Astron. Astrophys. Suppl. 1993, 102, 451-593.

127. Keller, S.C.; Wood, P.R.; Bessell, M.S. Be stars in and around young clusters in the Magellanic Clouds. Astron. Astrophys. Suppl. 1999, 134, 489-503. [CrossRef]

128. Keller, L.D.; Sloan, G.C.; Forrest, W.J.; Ayala, S.; D’Alessio, P.; Shah, S.; Calvet, N.; Najita, J.; Li, A.; Hartmann, L.; et al. PAH Emission from Herbig Ae/Be Stars. Astrophys. J. 2008, 684, 411-429. [CrossRef]

129. Peeters, E.; Hony, S.; Van Kerckhoven, C.; Tielens, A.G.G.M.; Allamandola, L.J.; Hudgins, D.M.; Bauschlicher, C.W. The rich 6 to $9 \mu \mathrm{m}$ spectrum of interstellar PAHs. Astron. Astrophys. 2002, 390, 1089-1113. [CrossRef]

130. Ruffle, P.M.E.; Kemper, F.; Jones, O.C.; Sloan, G.C.; Kraemer, K.E.; Woods, P.M.; Boyer, M.L.; Srinivasan, S.; Antoniou, V.; Lagadec, E.; et al. Spitzer infrared spectrograph point source classification in the Small Magellanic Cloud. Mon. Not. R. Astron. Soc. 2015, 451, 3504-3536. [CrossRef]

131. Whelan, D.G.; Lebouteiller, V.; Galliano, F.; Peeters, E.; Bernard-Salas, J.; Johnson, K.E.; Indebetouw, R.; Brandl, B.R. An In-depth View of the Mid-infrared Properties of Point Sources and the Diffuse ISM in the SMC Giant H II Region, N66. Astrophys. J. 2013, 771, 16. [CrossRef]

132. Kamath, D.; Wood, P.R.; Van Winckel, H. Optically visible post-AGB/RGB stars and young stellar objects in the Small Magellanic Cloud: Candidate selection, spectral energy distributions and spectroscopic examination. Mon. Not. R. Astron. Soc. 2014, 439, 2211-2270. [CrossRef]

133. Heydari-Malayeri, M. Discovery of a low mass B[e] supergiant in the Small magellanic Cloud. Astron. Astrophys. 1990, 234, 233.

134. Corral, L.J. LBV-Type Stars in M33. Astron. J. 1996, 112, 1450. [CrossRef]

135. Fabrika, S.; Sholukhova, O. A survey of blue- $\mathrm{H} \alpha$ objects in the galaxy M 33. Astron. Astrophys. Suppl. 1999, 140, 309-326. [CrossRef]

136. Kraus, M.; Cidale, L.S.; Arias, M.L.; Oksala, M.E.; Borges Fernandes, M. Discovery of the First B[e] Supergiants in M 31. Astrophys. J. Lett. 2014, 780, L10. [CrossRef]

137. Sholukhova, O.; Bizyaev, D.; Fabrika, S.; Sarkisyan, A.; Malanushenko, V.; Valeev, A. New luminous blue variables in the Andromeda galaxy. Mon. Not. R. Astron. Soc. 2015, 447, 2459-2467. [CrossRef]

138. Kourniotis, M.; Kraus, M.; Arias, M.L.; Cidale, L.; Torres, A.F. On the evolutionary state of massive stars in transition phases in M33. Mon. Not. R. Astron. Soc. 2018, 480, 3706-3717. [CrossRef]

139. Sholukhova, O.; Fabrika, S.; Valeev, A. Classification of LBV-Star Candidates in the Galaxy M31. In Stars: From Collapse to Collapse; Balega, Y.Y., Kudryavtsev, D.O., Romanyuk, I.I., Yakunin, I.A., Eds.; ASP Conference Series; Astronomical Society of the Pacific: San Francisco, CA, USA, 2017; Volume 510, p. 468.

140. Humphreys, R.M.; Davidson, K.; Grammer, S.; Kneeland, N.; Martin, J.C.; Weis, K.; Burggraf, B. Luminous and Variable Stars in M31 and M33. I. The Warm Hypergiants and Post-red Supergiant Evolution. Astrophys. J. 2013, 773, 46. [CrossRef]

141. Fabrika, S.; Sholukhova, O.; Becker, T.; Afanasiev, V.; Roth, M.; Sanchez, S.F. Crowded field 3D spectroscopy of LBV candidates in M 33. Astron. Astrophys. 2005, 437, 217-226. [CrossRef]

142. Humphreys, R.M.; Davidson, K.; Hahn, D.; Martin, J.C.; Weis, K. Luminous and Variable Stars in M31 and M33. V. The Upper HR Diagram. Astrophys. J. 2017, 844, 40. [CrossRef]

143. Gordon, M.S.; Humphreys, R.M.; Jones, T.J. Luminous and Variable Stars in M31 and M33. III. The Yellow and Red Supergiants and Post-red Supergiant Evolution. Astrophys. J. 2016, 825, 50. [CrossRef]

144. Stahl, O.; Wolf, B.; de Groot, M.; Leitherer, C. Atlas of hig-dispersion spectra of peculiar emission-line stars in the Magellanic Clouds. Astron. Astrophys. Suppl. 1985, 61, 237-258.

145. Sholukhova, O.; Fabrika, S.; Roth, M.; Becker, T. B 416-A B[e]-SUPERGIANT in Interacting Binary? Balt. Astron. 2004, 13, 156-158.

146. Sandage, A. The brightest stars in nearby galaxies. II. The color-magnitude diagram for the brightest red and blue stars in M101. Astron. J. 1983, 88, 1569-1578. [CrossRef]

147. Sandage, A. The brightest stars in nearby galaxies. III. The color-magnitude diagram for the brightest red and blue stars in M 81 and Holmberg IX. Astron. J. 1984, 89, 621-629. [CrossRef]

148. Sandage, A. The brightest stars in nearby galaxies. IV. The color-magnitude diagram for the brightest red and blue stars in NGC 2403. Astron. J. 1984, 89, 630-635. [CrossRef] 
149. Zickgraf, F.-J.; Humphreys, R.M. A Stellar Content Survey of NGC 2403 and M81. Astron. J. 1991, $102,113$. [CrossRef]

150. Grammer, S.; Humphreys, R.M. The Massive Star Population in M101. I. The Identification and Spatial Distribution of the Visually Luminous Stars. Astron. J. 2013, 146, 114. [CrossRef]

151. Humphreys, R.M. Studies of luminous stars in nearby galaxies. VII-The brightest blue stars in the spiral galaxies M101 and NGC 2403. Astrophys. J. 1980, 241, 598-601. [CrossRef]

152. Humphreys, R.M.; Aaronson, M. The Visually Brightest Early-Type Supergiants in the Spiral Galaxies NGC 2403, M81, and M101. Astron. J. 1987, 94, 1156, [CrossRef]

153. Sholukhova, O.N.; Fabrika, S.N.; Vlasyuk, V.V.; Dodonov, S.N. Spectroscopy of stars in the galaxy M 81. Astron. Lett. 1998, 24, 507-515.

154. Sholukhova, O.N.; Fabrika, S.N.; Vlasyuk, V.V. Spectroscopy of stars in the Galaxy NGC 2403. Astron. Lett. 1998, 24, 603-610.

155. Grammer, S.H.; Humphreys, R.M.; Gerke, J. The Massive Star Population in M101. III. Spectra and Photometry of the Luminous and Variable Stars. Astron. J. 2015, 149, 152. [CrossRef]

156. Humphreys, R.M.; Stangl, S.; Gordon, M.S.; Davidson, K.; Grammer, S.H. Luminous and Variable Stars in NGC 2403 and M81. Astron. J. 2019, 157, 22. [CrossRef]

157. Pustilnik, S.A.; Makarova, L.N.; Perepelitsyna, Y.A.; Moiseev, A.V.; Makarov, D.I. The extremely metal-poor galaxy DDO 68: The luminous blue variable, $\mathrm{H} \alpha$ shells and the most luminous stars. Mon. Not. R. Astron. Soc. 2017, 465, 4985-5002. [CrossRef]

158. Spetsieri, Z.T.; Bonanos, A.Z.; Kourniotis, M.; Yang, M.; Lianou, S.; Bellas-Velidis, I.; Gavras, P.; Hatzidimitriou, D.; Kopsacheili, M.; Moretti, M.I.; et al. Massive variable candidates with the Hubble Space Telescope. Astron. Astrophys. 2018, 618, A185. [CrossRef]

159. Spetsieri, Z.T.; Bonanos, A.Z.; Yang, M.; Kourniotis, M.; Hatzidimitriou, D. The HST Key Project galaxies NGC 1326A, NGC 1425 and NGC 4548: New variable stars and massive star population. Astron. Astrophys. 2019, 629, A3. [CrossRef]

160. Ababakr, K.M.; Oudmaijer, R.D.; Vink, J.S. A statistical spectropolarimetric study of Herbig Ae/Be stars. Mon. Not. R. Astron. Soc. 2017, 472, 854-868. [CrossRef]

161. Bik, A.; Thi, W.F. Evidence for an inner molecular disk around massive Young Stellar Objects. Astron. Astrophys. 2004, 427, L13-L16. [CrossRef]

162. Blum, R.D.; Barbosa, C.L.; Damineli, A.; Conti, P.S.; Ridgway, S. Accretion Signatures from Massive Young Stellar Objects. Astrophys. J. 2004, 617, 1167-1176. [CrossRef]

163. Ilee, J.D.; Wheelwright, H.E.; Oudmaijer, R.D.; de Wit, W.J.; Maud, L.T.; Hoare, M.G.; Lumsden, S.L.; Moore, T.J.T.; Urquhart, J.S.; Mottram, J.C. CO bandhead emission of massive young stellar objects: Determining disc properties. Mon. Not. R. Astron. Soc. 2013, 429, 2960-2973. [CrossRef]

164. Ilee, J.D.; Fairlamb, J.; Oudmaijer, R.D.; Mendigutía, I.; van den Ancker, M.E.; Kraus, S.; Wheelwright, H.E. Investigating the inner discs of Herbig Ae/Be stars with $\mathrm{CO}$ bandhead and Br $\gamma$ emission. Mon. Not. R. Astron. Soc. 2014, 445, 3723-3736. [CrossRef]

165. Ilee, J.D.; Oudmaijer, R.D.; Wheelwright, H.E.; Pomohaci, R. Blinded by the light: On the relationship between $\mathrm{CO}$ first overtone emission and mass accretion rate in massive young stellar objects. Mon. Not. R. Astron. Soc. 2018, 477, 3360-3368. [CrossRef]

166. Esteban, C.; Fernandez, M. S266: A ring nebula around a Galactic B[e] supergiant? Mon. Not. R. Astron. Soc. 1998, 298, 185-192. [CrossRef]

167. Gvaramadze, V.V.; Menten, K.M. Discovery of a parsec-scale bipolar nebula around MWC 349A. Astron. Astrophys. 2012, 541, A7. [CrossRef]

168. Cohen, M.; Bieging, J.H.; Dreher, J.W.; Welch, W.J. The binary system MWC 349. Astrophys. J. 1985, 292, 249-256. [CrossRef]

169. Marchiano, P.; Brandi, E.; Muratore, M.F.; Quiroga, C.; Ferrer, O.E.; García, L.G. The spectroscopic orbits and physical parameters of GG Carinae. Astron. Astrophys. 2012, 540, A91. [CrossRef]

170. Miroshnichenko, A.S.; Levato, H.; Bjorkman, K.S.; Grosso, M. Properties of galactic B[e] supergiants II. HDE 327083. Astron. Astrophys. 2003, 406, 673-683. [CrossRef]

171. Wolf, B.; Stahl, O. The absorption spectrum of the Be star MWC 300. Astron. Astrophys. 1985, 148, $412-416$. 
172. Miroshnichenko, A.S.; Levato, H.; Bjorkman, K.S.; Grosso, M.; Manset, N.; Men'shchikov, A.B.; Rudy, R.J.; Lynch, D.K.; Mazuk, S.; Venturini, C.C.; et al. Properties of galactic B[e] supergiants. III. MWC 300. Astron. Astrophys. 2004, 417, 731-743. [CrossRef]

173. Miroshnichenko, A.; Corporon, P. Revealing the nature of the B[e] star MWC 342. Astron. Astrophys. 1999, 349, 126-134.

174. Millour, F.; Chesneau, O.; Borges Fernandes, M.; Meilland, A.; Mars, G.; Benoist, C.; Thiébaut, E.; Stee, P.; Hofmann, K.-H.; Baron, F.; et al. A binary engine fuelling HD 87643's complex circumstellar environment. Determined using AMBER/VLTI imaging. Astron. Astrophys. 2009, 507, 317-326. [CrossRef]

175. Plets, H.; Waelkens, C.; Trams, N.R. The peculiar binary supergiant 3 Puppis. Astron. Astrophys. 1995, 293, 363-370.

176. Meilland, A.; Kanaan, S.; Borges Fernandes, M.; Chesneau, O.; Millour, F.; Stee, P.; Lopez, B. Resolving the dusty circumstellar environment of the A[e] supergiant HD 62623 with the VLTI/MIDI. Astron. Astrophys. 2010, 512, A73. [CrossRef]

177. de Wit, W.J.; Oudmaijer, R.D.; Vink, J.S. Dusty Blue Supergiants: News from High-Angular Resolution Observations. Adv. Astron. 2014, 2014, 270848. [CrossRef]

178. Hendry, M.A.; Smartt, S.J.; Skillman, E.D.; Evans, C.J.; Trundle, C.; Lennon, D.J.; Crowther, P.A.; Hunter, I. The blue supergiant Sher 25 and its intriguing hourglass nebula. Mon. Not. R. Astron. Soc. 2008, 388, 1127-1142. [CrossRef]

179. Smith, N.; Arnett, W.D.; Bally, J.; Ginsburg, A.; Filippenko, A.V. The ring nebula around the blue supergiant SBW1: Pre-explosion snapshot of an SN 1987A twin. Mon. Not. R. Astron. Soc. 2013, 429, 1324-1341. [CrossRef]

180. Smith, N.; Tombleson, R. Luminous blue variables are antisocial: Their isolation implies that they are kicked mass gainers in binary evolution. Mon. Not. R. Astron. Soc. 2015, 447, 598-617. [CrossRef]

181. Richardson, N.D.; Mehner, A. The 2018 Census of Luminous Blue Variables in the Local Group. Res. Notes Am. Astron. Soc. 2018, 2, 121. [CrossRef]

182. Langer, N.; Heger, A. B[e] Supergiants: What is Their Evolutionary Status? In B[e] Stars; Hubert, A.M., Jaschek, C., Eds.; Astrophysics and Space Science Library: Dordrecht, The Netherlands; Kluwer Academic Publishers: Boston, MA, USA, 1998; Volume 233, p. 235.

183. Pasquali, A.; Nota, A.; Langer, N.; Schulte-Ladbeck, R.E.; Clampin, M. R4 and Its Circumstellar Nebula: Evidence for a Binary Merger? Astron. J. 2000, 119, 1352-1358. [CrossRef]

184. Martin, J.C.; Humphreys, R.M. Multi-epoch BVRI Photometry of Luminous Stars in M31 and M33. Astron. J. 2017, 154, 81. [CrossRef]

185. Stanek, K.Z.; Kaluzny, J.; Krockenberger, M.; Sasselov, D.D.; Tonry, J.L.; Mateo, M. Detached Eclipsing Binaries and Cepheids. III. Variables in the Field M31C. Astron. J. 1999, 117, 2810-2830. [CrossRef]

186. Vilardell, F.; Ribas, I.; Jordi, C. Eclipsing binaries suitable for distance determination in the Andromeda galaxy. Astron. Astrophys. 2006, 459, 321-331. [CrossRef]

187. Bartlett, E.S.; Clark, J.S.; Negueruela, I. CI Camelopardalis: The first sgB[e]-high mass X-ray binary twenty years on: A supernova imposter in our own Galaxy? Astron. Astrophys. 2019, 622, A93. [CrossRef]

188. Filliatre, P.; Chaty, S. The Optical/Near-Infrared Counterpart of the INTEGRAL Obscured Source IGR J16318-4848: An sgB[e] in a High-Mass X-Ray Binary? Astrophys. J. 2004, 616, 469-484. [CrossRef]

189. Lau, R.M.; Kasliwal, M.M.; Bond, H.E.; Smith, N.; Fox, O.D.; Carlon, R.; Cody, A.M.; Contreras, C.; Dykhoff, D.; Gehrz, R.; et al. Rising from the Ashes: Mid-infrared Re-brightening of the Impostor SN 2010da in NGC 300. Astrophys. J. 2016, 830, 142. [CrossRef]

190. Lau, R.M.; Heida, M.; Kasliwal, M.M.; Walton, D.J. First Detection of Mid-infrared Variability from an Ultraluminous X-Ray Source Holmberg II X-1. Astrophys. J. Lett. 2017, 838, L17. [CrossRef]

191. Villar, V.A.; Berger, E.; Chornock, R.; Margutti, R.; Laskar, T.; Brown, P.J.; Blanchard, P.K.; Czekala, I.; Lunnan, R.; Reynolds, M.T. The Intermediate Luminosity Optical Transient SN 2010da: The Progenitor, Eruption, and Aftermath of a Peculiar Supergiant High-mass X-ray Binary. Astrophys. J. 2016, 830, 11. [CrossRef]

(c) 2019 by the author. Licensee MDPI, Basel, Switzerland. This article is an open access article distributed under the terms and conditions of the Creative Commons Attribution (CC BY) license (http://creativecommons.org/licenses/by/4.0/). 